\title{
CENTRAL SLICES OF THE REGULAR SIMPLEX
}

PhD Thesis 1996

by

Simon Peter Webb

University College London.

Supervised by Dr K.M.Ball. 
ProQuest Number: 10018677

All rights reserved

\title{
INFORMATION TO ALL USERS
}

The quality of this reproduction is dependent upon the quality of the copy submitted.

In the unlikely event that the author did not send a complete manuscript and there are missing pages, these will be noted. Also, if material had to be removed, a note will indicate the deletion.

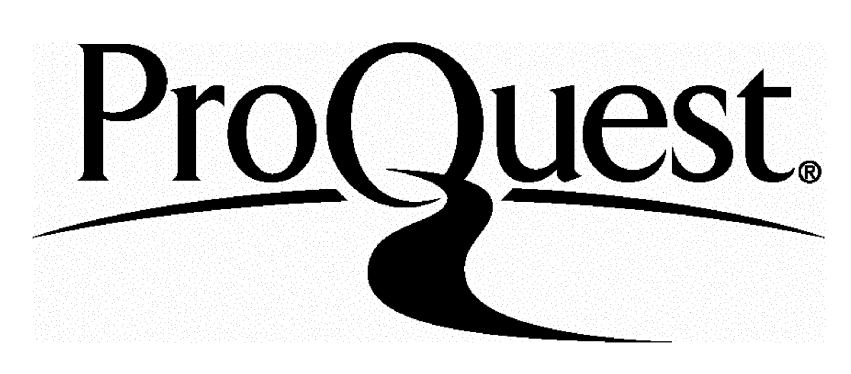

ProQuest 10018677

Published by ProQuest LLC(2016). Copyright of the Dissertation is held by the Author.

All rights reserved.

This work is protected against unauthorized copying under Title 17, United States Code. Microform Edition $\odot$ ProQuest LLC.

\author{
ProQuest LLC \\ 789 East Eisenhower Parkway \\ P.O. Box 1346 \\ Ann Arbor, MI 48106-1346
}




\begin{abstract}
In this thesis we investigate different methods of proving best upper bounds for the volumes of central sections of the regular $n$-dimensional simplex.

In Chapter 1 we show, using probabilistic methods, that the 1-codimensional central sections with maximal volume are exactly those sections that contain $n-1$ of the vertices of the simplex. The proof uses results about logarithmically concave functions on $R$. We note that there are both similarities and differences between this proof and that for the case of the $n$-dimensional cube, and we also give an intriguing reinterpretation of the result involving interpolation.

In Chapter 2 we examine the possibility of extending the 1-codimensional result of Chapter 1 to sections of any dimension. We show that the problem will reduce to a question about the position of the centroid of central slices of regular simplices in one dimension lower.

In Chapter 3 we show that the maximal 2-dimensional central slices of the regular simplex are those that contain 2 of the vertices. We prove this by obtaining best upper bounds on the volumes of maximal ellipsoids in central slices of the simplex. The proof involves making estimates on the determinants of matrices of the form $\sum_{i=1}^{n} z_{i} \otimes z_{i}$ where $\left(z_{i}\right)_{i=1}^{n}$ is a sequence of vectors in $R^{k}$.

Chapter 4 is a discussion of how our new results compare with those of P.Filliman, who gave conditions that must be satisfied by critical central sections (with respect to volume) of the regular simplex.
\end{abstract}




\section{Contents}

Acknowledgements $\quad \ldots \ldots \ldots \ldots \ldots \ldots \ldots \ldots \ldots \ldots \ldots \ldots \ldots \ldots \ldots$ page 4

Introduction $\quad \ldots \ldots \ldots \ldots \ldots \ldots \ldots \ldots \ldots \ldots \ldots \ldots \ldots \ldots \ldots \ldots$ page 5

Chapter $1 \quad \ldots \ldots \ldots \ldots \ldots \ldots \ldots \ldots \ldots \ldots \ldots \ldots \ldots \ldots \ldots$ page 11

Chapter $2 \quad \ldots \ldots \ldots \ldots \ldots \ldots \ldots \ldots \ldots \ldots \ldots \ldots \ldots \ldots \ldots \ldots$ page 23

Chapter $3 \quad \ldots \ldots \ldots \ldots \ldots \ldots \ldots \ldots \ldots \ldots \ldots \ldots \ldots$ page 38

Chapter $4 \quad \ldots \ldots \ldots \ldots \ldots \ldots \ldots \ldots \ldots \ldots \ldots \ldots \ldots \ldots \ldots \ldots$ page 65

References $\quad \ldots \ldots \ldots \ldots \ldots \ldots \ldots \ldots \ldots \ldots \ldots \ldots \ldots \ldots$ page 74 


\section{Acknowledgements}

I would like to thank my supervisor Keith Ball for all his help over the past three years. His advice, encouragement and endless patience have been absolutely invaluable.

Also, I would like to thank all the staff and postgraduate students in the mathematics departments of University College London and Texas A \& M University for their moral support and many helpful discussions, and the EPSRC (formerly the SERC) for their financial support.

Finally, I would like to thank my parents for always believing in me, and for supporting me throughout my university education. 


\section{Introduction}

In this work we examine the problem of finding maximal central slices of the regular $n$-simplex. Apart from the intrinsic geometric appeal of the problem, the question is interesting because results about volumes of sections provide estimates for densities of sums of i.i.d. random variables. Moreover, the methods used here involve estimating determinants of matrices of the form $\sum_{i=1}^{n} z_{i} \otimes z_{i}$ for a sequence of vectors $\left(z_{i}\right)_{i=1}^{n}$ and this is a problem which arises in other areas of mathematics.

In 1979 Hensley [9] showed that if $Q_{n}=\left[-\frac{1}{2}, \frac{1}{2}\right]^{n}$ is the central unit cube in $R^{n}$ and $H$ is an $(n-1)$-dimensional subspace of $R^{n}$ then $\operatorname{Vol}\left(H \cap Q_{n}\right)$ lies between 1 and 5. At about the same time, Vaaler [12] showed that the lower bound of 1 for the volume holds for sections of any dimension. Hensley conjectured that the best upper bound for the volume of central sections of the cube is $\sqrt{2}$, and this result was proved by Ball [1] in 1986. In [2] Ball generalised this result, showing that if $H$ is a $k$-codimensional subspace, $1 \leq k \leq n-1$, of $R^{n}$ then,

$$
\operatorname{Vol}\left(H \bigcap Q_{n}\right) \leq(\sqrt{2})^{k}
$$

This upper bound is the best possible for every $k$ and is attained for some $H$ if $n \geq 2 k$. 
For the $\ell_{p}$ ball,

$$
B_{p}^{n}=\left\{\mathrm{x}=\left(x_{1}, \ldots, x_{n}\right) \in R^{n}: \sum_{i=1}^{n}\left|x_{i}\right|^{p} \leq 1\right\}
$$

Meyer and Pajor [10] showed that for $1 \leq p \leq q \leq \infty$, if $H$ is a $k$-dimensional subspace of $R^{n}, 1 \leq k \leq n-1$, then

$$
\frac{\operatorname{Vol}\left(H \cap B_{p}^{n}\right)}{\operatorname{Vol}\left(B_{p}^{k}\right)} \leq \frac{\operatorname{Vol}\left(H \cap B_{q}^{n}\right)}{\operatorname{Vol}\left(B_{q}^{k}\right)}
$$

Taking $q=2$ gives us that the $k$-dimensional central sections of $B_{p}^{n}$ have volume at most that of $B_{p}^{k}$ when $1 \leq p \leq 2$. Taking $p=2$ gives us that the $k$-dimensional central sections of $B_{q}^{n}$ have volume at least that of $B_{q}^{k}$ when $q \geq 2$. The case $p=2, q=\infty$ is exactly Vaaler's result.

It is an immediate consequence of the Brunn-Minkowski inequality, namely, for measurable sets $A, B \subset R^{k}$,

$$
\operatorname{Vol}\left(\frac{1}{2} A+\frac{1}{2} B\right)^{\frac{1}{k}} \geq \frac{1}{2} \operatorname{Vol}(A)^{\frac{1}{k}}+\frac{1}{2} \operatorname{Vol}(B)^{\frac{1}{k}}
$$

that if $X$ is a convex, centrally symmetric body in $R^{n}$ and $P$ is a $k$-dimensional section of $X$, then the volume of $P$ is at most the volume of the $k$-dimensional section of $X$ parallel to $P$ and containing the origin. This is because if $H$ is a $k$-dimensional subspace and a a point in $R^{n}$ then

$$
\begin{aligned}
& (H+\mathbf{a}) \bigcap X=A \\
& (H-\mathbf{a}) \bigcap X=-A
\end{aligned}
$$

and by the convexity of $\mathrm{X}$,

$$
H \bigcap X \supseteq \frac{1}{2}(A-A)
$$


so,

$$
\begin{aligned}
\operatorname{Vol}(H \bigcap X)^{\frac{1}{k}} & \geq \operatorname{Vol}\left(\frac{1}{2}(A-A)\right)^{\frac{1}{k}} \\
& \geq \frac{1}{2} \operatorname{Vol}(A)^{\frac{1}{k}}+\frac{1}{2} \operatorname{Vol}(-A)^{\frac{1}{k}} \\
& =\operatorname{Vol}((H+\mathbf{a}) \bigcap X)^{\frac{1}{k}}
\end{aligned}
$$

This means that the search for an upper bound on the volume of sections of a centrally symmetric body may be restricted to central sections.

However this is not the case for nonsymmetric bodies, the most important of which is the simplex. It is easy to see that the longest line segment in a plane triangle is one of the sides. Brands and Laman [5] and Eggleston [7] showed in 1963 that the plane section of a tetrahedron (not necessarily a regular tetrahedron ) with largest area is one of the faces. Walkup [13] showed that the natural generalisation of these results to higher dimensions does not hold, by proving that there is a 5 dimensional simplex with a 4-dimensional cross section of greater volume than any of the 4-dimensional faces of the simplex.

In [3] Ball,in response to questions from M.Klamkin and C.Greene, showed that if $S$ is a regular $n$-dimensional simplex of internal radius 1 , then for each $1 \leq k \leq n$, the $k$-dimensional ellipsoids of maximal volume contained in $S$ are Euclidean balls of radius $\sqrt{\frac{n(n+1)}{k(k+1)}}$ which lie in the $k$-dimensional faces of $S$.

The volume ratio of an $n$-dimensional convex body $C$ is defined,

$$
\operatorname{vr}(C)=\left(\frac{\operatorname{Vol}(C)}{\operatorname{Vol}(\varepsilon)}\right)^{\frac{1}{n}}
$$

where $\varepsilon$ is the ellipsoid of maximal volume contained in $C$. Ball proved in [4], 
that the simplex has largest volume ratio, so the result in [3] implies that the $k$ dimensional sections of a regular $n$-simplex with largest volume are its $k$-dimensional faces.

Since results of this type, about volumes of sections, provide estimates for densities of sums of i.i.d. random variables, possibly a more interesting problem is that of finding central sections of the regular simplex with maximal volume since this will involve random variables with mean zero.

In Chapter 1 we prove, using probabilistic methods that the 1-codimensional central sections of largest volume are those which are perpendicular to an edge of the simplex. We note that there are similarities to the proof for the cube in [1], but that the difficulties occur in different places and require a discussion of logarithmically concave functions. A second proof of the result is given, using only the abstract theory of logarithmically concave functions.

In Chapter 2 we discuss the problem of extending the 1-codimensional proof to other dimensions of section by using induction on the codimension of the slice. We reduce the problem to a question concerning the position of the centroid of central slices, of regular $(n-1)$-dimensional simplices.

In Chapter 3 we examine maximal ellipsoids in central slices of the regular simplex and, using the volume ratio result in [4] derive a solution to the maximal slice problem for 2-dimensional slices. The proof involves estimating the determinants of $k \times k$ matrices of the form $\sum_{i=1}^{n+1} z_{i} \otimes z_{i}$ where $\left(z_{i}\right)_{i=1}^{n+1}$ is a sequence of vectors in $R^{k}$ satisfying certain conditions, by firstly making estimates on the length of $z_{i} \wedge z_{j}$ 
for each pair of vectors and then summing over all pairs $i \neq j$.

In Chapter 4 we compare our results to those of Filliman [8], who gave conditions that must be satisfied by critical sections.

Experience of these types of geometric inequalities tells us that the only easy cases are those of 1-dimensional slices. This is certainly true for central slices of the regular simplex. The following proof demonstrates the simplicity of the 1dimensional case and introduces a useful characterisation of the regular simplex in terms of its boundary functionals.

Let $S$ be the regular simplex in $R^{n}$ :

$$
S=\left\{x \in R^{n}: \quad<x, u_{i}>\leq 1 \quad i=1, \ldots, n+1\right\}
$$

where $\left(u_{i}\right)_{i=1}^{n+1}$ is a sequence of unit vectors satisfying

$$
\sum_{i=1}^{n+1} u_{i}=0
$$

and

$$
\frac{n}{n+1} \sum_{i=1}^{n+1} u_{i} \otimes u_{i}=I_{n}
$$

where $I_{n}$ denotes the identity on $R^{n}$.

Let $x$ be a point on the boundary of $S$ and $r \in R$ such that $-r x \in S$. Then the line segment $[-r x, x]$ passes through the centroid of $S$, and we show that the length of this line is at most $n+1$.

We have $1 \leq\|x\| \leq n$ and for every $1 \leq i \leq n+1$,

$$
-\frac{1}{r} \leq<x, u_{i}>\leq 1
$$


so,

$$
\sum_{i=1}^{n+1}\left(1-<x, u_{i}>\right)\left(<x, u_{i}>+\frac{1}{r}\right) \geq 0
$$

Expanding this gives,

$$
\frac{n+1}{r}+\left(1-\frac{1}{r}\right) \sum_{i=1}^{n+1}<x, u_{i}>-\sum_{i=1}^{n+1}<x, u_{i}>^{2} \geq 0
$$

that is,

$$
\sum_{i=1}^{n+1}<x, u_{i}>^{2} \leq \frac{n+1}{r}
$$

or,

$$
\frac{n+1}{n}\|x\|^{2} \leq \frac{n+1}{r}
$$

and so,

$$
r\|x\|^{2} \leq n
$$

Therefore the length of the line segment $[-r x, x]$ is

$$
(1+r)\|x\| \leq \frac{n}{\|x\|}+\|x\|
$$

and by the convexity of the function $t \rightarrow \frac{n}{t}+t$ on $[1, n]$ we have,

$$
(1+r)\|x\| \leq n+1
$$

So any line through the centroid of the regular simplex $S$ has length at most $n+1$, and this upper bound is attained if and only if the line passes through a vertex.

The above characterisation of the $n$-simplex, in terms of a sequence of unit vectors $\left(u_{i}\right)_{i=1}^{n+1}$ in $R^{n}$, is particularly useful in Chapter 3 . 


\section{Chapter 1}

In this chapter we will show that the $(n-1)$-dimensional slices of a regular $n$-simplex passing through the centroid of largest volume are exactly the slices that contain $n-1$ of the vertices. We will describe two proofs of this statement, discuss the merits of each, and note that the result has an intriguing reinterpretation involving interpolation.

Since the $n$-simplex is not easily represented in $R^{n}$ we will consider the natural embedding in $R^{n+1}$. For $t>0$ the set

$$
S_{t}=\left\{\mathbf{x}=\left(x_{1}, \ldots, x_{n+1}\right) \in R^{n+1}: x_{i} \geq 0 \quad i=1, \ldots, n+1 \quad, \quad \sum_{i=1}^{n+1} x_{i}=t\right\}
$$

is the regular $n$-dimensional simplex formed by taking the convex hull of $\left\{t e_{j}\right\}_{j=1}^{n+1}$ where $\left\{\mathbf{e}_{j}\right\}_{j=1}^{n+1}$ are the standard basis vectors in $R^{n+1}$.

Let $\mathbf{a}=\left(a_{1}, \ldots, a_{n+1}\right)$ be a unit vector in $R^{n+1}$ and $H$ the subspace of $R^{n+1}$ perpendicular to a. If $\sum_{i=1}^{n+1} a_{i}=0$ then $H \cap S_{t}$ is an $(n-1)$-dimensional slice of $S_{t}$ passing through the centroid. Our aim here will be to estimate $\operatorname{Vol}_{n-1}\left(H \cap S_{1}\right)$ using probabilistic methods. 
Define functions $f: R \rightarrow[0, \infty)$ and $F: R^{n+1} \rightarrow[0, \infty)$ by

$$
\begin{gathered}
f(x)= \begin{cases}e^{-x} & \text { if } x \geq 0 \\
0 & \text { otherwise }\end{cases} \\
F(\mathbf{x})=\prod_{j=1}^{n+1} f\left(x_{j}\right)
\end{gathered}
$$

Let $H$ and $\mathbf{a}$ be as above with $\sum_{i=1}^{n+1} a_{j}=0$.

For a fixed $t>0, F$ is constant on $S_{t}$ and

$$
\operatorname{Vol}_{n-1}\left(H \bigcap S_{t}\right)=t^{n-1} \operatorname{Vol}_{n-1}\left(H \bigcap S_{1}\right)
$$

so a change of coordinates gives

$$
\begin{aligned}
\int_{H} F d V o l_{H} & =\int_{H} \prod_{j=1}^{n+1} f\left(x_{j}\right) d \operatorname{Vol}_{H} \\
& =\int_{0}^{\infty} e^{-s \sqrt{n+1}} \operatorname{Vol}_{n-1}\left(H \bigcap S_{s \sqrt{n+1}}\right) d s \\
& =\int_{0}^{\infty} e^{-s \sqrt{n+1}}(s \sqrt{n+1})^{n-1} \operatorname{Vol}_{n-1}\left(H \bigcap S_{1}\right) d s \\
& =(n-1) !(n+1)^{-\frac{1}{2}} \operatorname{Vol}_{n-1}\left(H \bigcap S_{1}\right)
\end{aligned}
$$

that is

$$
V o l_{n-1}\left(H \bigcap S_{1}\right)=\frac{\sqrt{n+1}}{(n-1) !} \int_{H} F d V o l_{H}
$$

where $V o l_{H}$ denotes Lebesgue measure on $H$.

We wish to estimate the integral $\int_{H} F d V o l_{H}$ which may be interpreted as the value at zero of the density of a certain random variable as follows. Consider a sequence of i.i.d. random variables $\left\{X_{j}\right\}_{j=1}^{n+1}$ each with density $f$. For any real sequence $\left\{a_{j}\right\}_{j=1}^{n+1}$ with $\sum_{i=1}^{n+1} a_{j}^{2}=1$ the density of the random variable $\sum_{i=1}^{n+1} a_{j} X_{j}$ can be written

$$
G(t)=\int_{H+t \mathbf{a}} F(\mathbf{x}) d V o l_{H}
$$


so

$$
G(0)=\int_{H} F d V o l_{H}
$$

So our aim is to estimate $G(0)$. In these terms, our theorem becomes, $G(0) \leq \frac{1}{\sqrt{2}}$, with equality if and only if $n-1$ of the $a_{j}$ are zero. The proof of the theorem will divide into two parts, the first for $\left|a_{j}\right| \leq \frac{1}{\sqrt{2}}$ for each $1 \leq j \leq n+1$ and the second for the case $\left|a_{j}\right|>\frac{1}{\sqrt{2}}$ for some $1 \leq j \leq n+1$. This is analogous to the proof for the cube in [1]. We use the same approach for the first part and apply an easier estimate than was required in [1], but for the second part , the easy part for the cube, we need a different method. We now introduce some preliminary results concerning logarithmically concave functions.

\section{Preliminary Results}

Definition 1.1 A function $f: R^{n} \rightarrow R$ is called logarithmically concave if for any $\mathbf{x}, \mathbf{y} \in R^{n}$ and $0<\lambda<1, f(\lambda \mathbf{x}+(1-\lambda) \mathbf{y}) \geq f(\mathbf{x})^{\lambda} f(\mathbf{y})^{1-\lambda}$.

It is well known that if $f: R^{n} \rightarrow[0, \infty)$ is $\log$ concave, then so is each of its marginals, e.g. the function $g: R^{n-1} \rightarrow[0, \infty)$ defined by

$$
g\left(x_{1}, \ldots, x_{n-1}\right)=\int f\left(x_{1}, \ldots, x_{n}\right) d x_{n}
$$

For a proof of this see, for example, [11]. Since products of log concave functions are log concave, it follows that log concavity is preserved under convolution.

Results of the following type have been proved independently by many authors. They seem to go back to Schur and Ostrowski or even further. A new twist is provided here by the fact that $G$ is not assumed to be decreasing. 
Lemma 1.2 Let $G: R \rightarrow[0, \infty)$ be a logarithmically concave function and $k>0$. Then

$$
G(0)^{k} \int_{0}^{\infty} G(x) x^{k} d x \leq \Gamma(k+1)\left(\int_{0}^{\infty} G(x) d x\right)^{k+1}
$$

\section{Proof}

Without loss of generality we may assume that $G(0)=1$ and that $\int_{0}^{\infty} G(x) d x=1$.

So write $G(x)=e^{-\phi(x)}$ where $\phi: R \rightarrow R$ is convex and $\phi(0)=0$. We need to prove that $\int_{0}^{\infty} G(x) x^{k} d x \leq \Gamma(k+1)$.

Note that by the convexity of $\phi$, if $u, v \in R$ with $0 \leq u \leq v$ then $\phi(u) \leq \frac{u}{v} \phi(v)$.

Let $x \in[0, \infty)$. If $x \leq \phi(x)$ then

$$
\begin{aligned}
\int_{x}^{\infty} G(v) d v & \leq \int_{x}^{\infty} e^{-v \frac{\phi(x)}{x}} d v \\
& =\frac{x}{\phi(x)} e^{-\phi(x)} \\
& \leq e^{-x}=\int_{x}^{\infty} e^{-v} d v
\end{aligned}
$$

If $x \geq \phi(x)$ then

$$
\begin{aligned}
\int_{0}^{x} G(u) d u & \geq \int_{0}^{x} e^{-u \frac{\phi(x)}{x}} d u \\
& =\frac{x}{\phi(x)} \int_{0}^{\phi(x)} e^{-u} d u \\
& \geq \int_{0}^{x} e^{-u} d u
\end{aligned}
$$

because $\frac{1}{x} \int_{0}^{x} e^{-u} d u$ is a decreasing function of $x$.

Since $\int_{0}^{\infty} G(v) d v=\int_{0}^{\infty} e^{-v} d v$ we have for any $x \in[0, \infty)$,

$$
\int_{x}^{\infty} G(v) d v \leq \int_{x}^{\infty} e^{-v} d v=e^{-x}
$$


hence

$$
\int_{0}^{\infty} x^{k-1} \int_{x}^{\infty} G(v) d v \leq \int_{0}^{\infty} x^{k-1} e^{-x} d x=\Gamma(k)
$$

that is

$$
\int_{0}^{\infty} G(v) v^{k} d v \leq \Gamma(k+1)
$$

Lemma 1.3 Let $f$ be a positive logarithmically concave function on $R$. Then

$$
\left(\int_{0}^{\infty} f(x) e^{-x} d x\right)\left(\int_{0}^{\infty} f(x)(1+x) d x\right) \leq\left(\int_{0}^{\infty} f(x) d x\right)^{2}
$$

\section{Proof}

Define $G(t)=e^{t} \int_{t}^{\infty} e^{-u} f(u) d u$. Then $G$ is the convolution of two log concave functions and so is log concave. We have

$$
\begin{aligned}
\int_{0}^{\infty} G(t) t d t & =\int_{0}^{\infty} f(u)\left(u-1+e^{-u}\right) d u \\
\int_{0}^{\infty} G(t) d t & =\int_{0}^{\infty} f(u)\left(1-e^{-u}\right) d u
\end{aligned}
$$

Applying Lemma 1.2 with $k=1$ and rearranging gives the desired result. 


\section{The Main Theorem}

Theorem 1.4 Let $S$ be a regular $n$-dimensional simplex with edges of length $\sqrt{2}$. Then the volume of any 1-codimensional slice of $S$ passing through the centroid is at most $\frac{\sqrt{n+1}}{(n-1) ! \sqrt{2}}$. This upper bound is attained when and only when the slice contains $n-1$ vertices of $S$.

\section{Proof}

We need to show, with $F$ and $H$ as above, that $\int_{H} F d V o l_{H} \leq \frac{1}{\sqrt{2}} \quad$ for any vector $\mathbf{a}=\left(a_{1}, \ldots, a_{n+1}\right)$ with $\sum_{j=1}^{n+1} a_{j}^{2}=1$ and $\sum_{j=1}^{n+1} a_{j}=0$

The proof splits into two cases :

CASE $1\left|a_{j}\right| \leq \frac{1}{\sqrt{2}} \quad j=1, \ldots, n+1$

Denoting by $\Phi_{Y}$ the characteristic function of a random variable $Y$,

$$
\begin{aligned}
\Phi_{\sum_{j=1}^{n+1} a_{j} X_{j}}(t) & =\prod_{j=1}^{n+1} \Phi_{X_{j}}\left(a_{j} t\right) \\
& =\prod_{j=1}^{n+1} \frac{1}{1+i a_{j} t}
\end{aligned}
$$

The standard Fourier inversion formula gives

$$
G(s)=\frac{1}{2 \pi} \int_{-\infty}^{\infty} \prod_{j=1}^{n+1} \frac{1}{1+i a_{j} t} e^{i s t} d t
$$

Hence

$$
\int_{H} F d V o l_{H}=G(0)=\frac{1}{2 \pi} \int_{-\infty}^{\infty} \prod_{j=1}^{n+1} \frac{1}{1+i a_{j} t} d t
$$

We may now assume that all the $a_{j}$ are non-zero or the formula would reduce to the $(n-1)$-dimensional case, so

$$
\int_{H} F d V o l_{H}=\frac{1}{2 \pi} \int_{-\infty}^{\infty} \prod_{j=1}^{n+1} \frac{1}{1+i a_{j} t} d t
$$




$$
\leq \frac{1}{2 \pi} \int_{-\infty}^{\infty} \prod_{j=1}^{n+1}\left|\frac{1}{1+i a_{j} t}\right| d t
$$

Applying Hölder's inequality using the fact $\sum_{j=1}^{n+1} a_{j}^{2}=1$

$$
\begin{aligned}
\int_{H} F d V o l_{H} & \leq \frac{1}{2 \pi} \prod_{j=1}^{n+1}\left(\int_{-\infty}^{\infty}\left|\frac{1}{1+i a_{j} t}\right|^{a_{j}^{-2}} d t\right)^{a_{j}^{2}} \\
& =\frac{1}{2 \pi} \prod_{j=1}^{n+1}\left(\int_{-\infty}^{\infty}\left(\frac{1}{1+a_{j}^{2} t^{2}}\right)^{\frac{1}{2} a_{j}^{-2}} d t\right)^{a_{j}^{2}}
\end{aligned}
$$

Now using the fact that for $\alpha>0$ and $0<\gamma \leq 1,(1+\alpha)^{\gamma} \leq 1+\alpha \gamma$ we obtain the inequality

$$
\left(\frac{1}{1+\alpha \beta}\right)^{\frac{1}{2 \beta}} \leq \frac{1}{1+\frac{1}{2} \alpha}
$$

for $\alpha>0,0<\beta \leq \frac{1}{2}$, with equality only if $\beta=\frac{1}{2}$, so that

$$
\begin{aligned}
\int_{H} F d V o l_{H} & \leq \frac{1}{2 \pi} \prod_{j=1}^{n+1}\left(\int_{-\infty}^{\infty} \frac{1}{1+\frac{1}{2} t^{2}} d t\right)^{a_{j}^{2}} \\
& =\frac{1}{2 \pi} \prod_{j=1}^{n+1}(\sqrt{2} \pi)^{a_{j}^{2}} \\
& =\frac{1}{\sqrt{2}}
\end{aligned}
$$

with equality if and only if $n-1$ of the $a_{j}$ are zero.

CASE 2 For some $1 \leq j \leq n+1,\left|a_{j}\right|>\frac{1}{\sqrt{2}}$.

Without loss of generality assume $a_{1}>\frac{1}{\sqrt{2}}$. As we noticed earlier, $\int_{H+t_{\mathrm{a}}} F d V o l_{H}$ is the density of the random variable $\sum_{j=1}^{n+1} a_{j} X_{j}$ and so can be written as $(h * g)(t)$ the convolution of $h$, the density of the random variable $a_{1} X_{1}$, and $g$, the density 
of $\sum_{j=2}^{n+1} a_{j} X_{j}$. We need to estimate $(h * g)(0)$.

$$
\begin{aligned}
(h * g)(0) & =\int_{-\infty}^{\infty} h(x) g(-x) d x \\
& =\int_{0}^{\infty} \frac{1}{a_{1}} e^{-a_{1}^{-1} x} g(-x) d x \\
& =\int_{0}^{\infty} e^{-y} g\left(-a_{1} y\right) d y
\end{aligned}
$$

We shall estimate this using Lemma 1.3 so we need to know $\int_{0}^{\infty} y g\left(-a_{1} y\right) d y$ and $\int_{0}^{\infty} g\left(-a_{1} y\right) d y$. Since $g$ is positive, using $\mathbf{E}$ to denote expectation,

$$
\begin{aligned}
\int_{0}^{\infty} y g\left(-a_{1} y\right) d y & =-\int_{-\infty}^{0} \frac{y}{a_{1}^{2}} g(y) d y \\
& \geq-\frac{1}{a_{1}^{2}} \int_{-\infty}^{\infty} y g(y) d y \\
& =-\frac{1}{a_{1}^{2}} \mathbf{E}\left(\sum_{j=2}^{n+1} a_{j} X_{j}\right) \\
& =-\frac{1}{a_{1}^{2}} \sum_{j=2}^{n+1} a_{j} \mathbf{E}\left(X_{j}\right) \\
& =-\frac{1}{a_{1}^{2}} \sum_{j=2}^{n+1} a_{j} \\
& =\frac{1}{a_{1}} \\
\int_{0}^{\infty} g\left(-a_{1} y\right) d y & =\frac{1}{a_{1}} \int_{-\infty}^{0} g(y) d y \\
& \leq \frac{1}{a_{1}}
\end{aligned}
$$

As a convolution of $\log$ concave functions, $g$ is also log concave and so applying Lemma 1.3

$$
\begin{aligned}
\int_{0}^{\infty} e^{-y} g\left(-a_{1} y\right) d y & \leq \frac{\left(\int_{0}^{\infty} g\left(-a_{1} y\right) d y\right)^{2}}{\int_{0}^{\infty} g\left(-a_{1} y\right) d y+\int_{0}^{\infty} y g\left(-a_{1} y\right) d y} \\
& \leq \frac{\left(\int_{0}^{\infty} g\left(-a_{1} y\right) d y\right)^{2}}{\int_{0}^{\infty} g\left(-a_{1} y\right) d y+\frac{1}{a_{1}}}
\end{aligned}
$$




$$
\begin{aligned}
& \leq \frac{\left(\frac{1}{a_{1}}\right)^{2}}{\frac{1}{a_{1}}+\frac{1}{a_{1}}} \\
& =\frac{1}{2 a_{1}} \\
& <\frac{1}{\sqrt{2}}
\end{aligned}
$$

\section{Remarks}

Using contour integration we can evaluate the integral

$$
\frac{1}{2 \pi} \int_{-\infty}^{\infty} \prod_{j=1}^{n+1} \frac{1}{1+i a_{j} t} d t
$$

explicitly as

$$
\frac{1}{2} \sum_{k=1}^{n+1} \frac{1}{\left|a_{k}\right|} \prod_{j \neq k} \frac{a_{k}}{a_{k}-a_{j}}
$$

This expression is more or less impossible to estimate as it stands, but in view of Theorem 1.4 we have

Corollary 1.5 Let $\left\{a_{j}\right\}_{j=1}^{n+1}$ be a sequence of distinct non-zero real numbers with $\sum_{j=1}^{n+1} a_{j}^{2}=1$ and $\sum_{j=1}^{n+1} a_{j}=0$. Then

$$
0<\sum_{k=1}^{n+1} \frac{1}{\left|a_{k}\right|} \prod_{j \neq k} \frac{a_{k}}{a_{k}-a_{j}} \leq \sqrt{2}
$$

with equality on the right hand side exactly when $n=1$.

If we write $b_{j}=a_{j}^{-1}$ for each $1 \leq j \leq n+1$ and define

$$
H(x)=\sum_{k=1}^{n+1}\left|b_{k}\right| \prod_{j \neq k} \frac{b_{j}-x}{b_{j}-b_{k}}
$$


then $H\left(b_{k}\right)=\left|b_{k}\right|$ for all $1 \leq k \leq n+1$ and so $H$ is the polynomial of degree $\mathrm{n}$ interpolating $f(x)=|x|$ at $b_{1}, \ldots, b_{n+1}$. So Corollary 1.5 can be reformulated in the following way,

Corollary 1.6 Let $\left\{b_{j}\right\}_{j=1}^{n+1}$ be a sequence of distinct non-zero real numbers with $\sum_{j=1}^{n+1} b_{j}^{-1}=0$ and $H(x)$ the polynomial of degree $n$ interpolating $f(x)=|x|$ at $b_{1}, \ldots, b_{n+1}$. Then

$$
0<H(0) \leq \sqrt{\frac{2}{\sum_{j=1}^{n+1} b_{j}^{-2}}}
$$

We will now give a second proof of Theorem 1.4 using only the abstract theory of $\log$ concave functions. This, although simpler than the first proof uses a variant of Lemma 1.2 , and so is not an approach one could use in the problem of the largest $k$-dimensional slice for general $k$.

Lemma 1.7 Let $f: R \rightarrow[0, \infty)$ be a logarithmically concave function and suppose that $\int_{-\infty}^{\infty} f(x) x d x=0$. Then

$$
f(0)^{2} \int_{-\infty}^{\infty} f(x) x^{2} d x \leq \frac{1}{2}\left(\int_{-\infty}^{\infty} f(x) d x\right)^{3}
$$

\section{Proof}

Define functions $H_{1}$ and $H_{2}$ as follows,

$$
\begin{aligned}
& H_{1}(x)=\frac{1}{f(x)} \int_{x}^{\infty} f(t) d t \\
& H_{2}(x)=\frac{1}{f(x)} \int_{-\infty}^{x} f(t) d t
\end{aligned}
$$


whenever $f(x)>0$.

We claim that $H_{1}$ is a decreasing function and $H_{2}$ is an increasing one, so

$$
f(0) \int_{0}^{\infty} x \int_{x}^{\infty} f(t) d t d x \leq \int_{0}^{\infty} x f(x) \int_{0}^{\infty} f(t) d t d x
$$

that is

$$
f(0) \int_{0}^{\infty} x^{2} f(x) d x \leq 2 \int_{0}^{\infty} x f(x) d x \int_{0}^{\infty} f(x) d x
$$

and similarly,

$$
f(0) \int_{-\infty}^{0} x^{2} f(x) d x \leq 2 \int_{-\infty}^{0}-x f(x) d x \int_{-\infty}^{0} f(x) d x
$$

Assume without loss of generality that

$$
\int_{0}^{\infty} f(x) d x \leq \frac{1}{2} \int_{-\infty}^{\infty} f(x) d x .
$$

Since we have $\int_{0}^{\infty} x f(x) d x=\int_{-\infty}^{0}-x f(x) d x$, adding (1.1) and (1.2) gives

$$
f(0) \int_{-\infty}^{\infty} x^{2} f(x) d x \leq 2 \int_{0}^{\infty} x f(x) d x \int_{-\infty}^{\infty} f(x) d x
$$

Applying Lemma 1.2 with $k=1$, we have

$$
f(0) \int_{0}^{\infty} f(x) x d x \leq\left(\int_{0}^{\infty} f(x) d x\right)^{2}
$$

and so from (1.3) we get

$$
\begin{aligned}
f(0)^{2} \int_{-\infty}^{\infty} x^{2} f(x) d x & \leq 2\left(\int_{0}^{\infty} f(x) d x\right)^{2} \int_{-\infty}^{\infty} f(x) d x \\
& \leq 2\left(\frac{1}{2} \int_{-\infty}^{\infty} f(x) d x\right)^{2} \int_{-\infty}^{\infty} f(x) d x \\
& =\frac{1}{2}\left(\int_{-\infty}^{\infty} f(x) d x\right)^{3}
\end{aligned}
$$


which is the conclusion of the lemma.

We now show $H_{1}$ is a decreasing function. Writing $f(x)=e^{-\phi(x)}$ where $\phi$ is a convex function, we have that for any $x<y$ and $s \geq 0$,

$$
\phi(s+y)-\phi(s+x) \geq \phi(y)-\phi(x)
$$

hence

$$
\begin{aligned}
e^{-\phi(s+x)+\phi(x)} & \geq e^{-\phi(s+y)+\phi(y)} \\
\int_{0}^{\infty} e^{-\phi(s+x)+\phi(x)} d s & \geq \int_{0}^{\infty} e^{-\phi(s+y)+\phi(y)} d s \\
e^{\phi(x)} \int_{x}^{\infty} e^{-\phi(t)} d t & \geq e^{\phi(y)} \int_{y}^{\infty} e^{-\phi(t)} d t
\end{aligned}
$$

that is $H_{1}(x) \geq H_{1}(y)$. Similarly we may show $H_{2}$ is increasing which completes the proof.

Since the function $G(t)=\int_{H+t \mathbf{a}} F(\mathbf{x}) d V o l_{H}$ is a log concave density and

$$
\begin{aligned}
\int_{-\infty}^{\infty} G(t) t d t & =\mathrm{E}\left(\sum_{j=1}^{n+1} a_{j} X_{j}\right)=\sum_{j=1}^{n+1} a_{j}=0 \\
\int_{-\infty}^{\infty} G(t) t^{2} d t & =\operatorname{variance}\left(\sum_{j=1}^{n+1} a_{j} X_{j}\right)=\sum_{j=1}^{n+1} a_{j}^{2}=1
\end{aligned}
$$

we may now apply Lemma 1.7 to $G$ to obtain

$$
G(0)^{2} \leq \frac{1}{2}
$$

so,

$$
G(0) \leq \frac{1}{\sqrt{2}}
$$

proving Theorem 1.4. 


\section{Chapter 2}

In this chapter we will discuss the problem of generalising the 1-codimensional result of Chapter 1 to the case of $k$-dimensional slices for any $1 \leq k \leq n-1$.

We conjecture that the correct generalisation of the 1-codimensional result is, Conjecture 2.1 Let $S$ be a regular $n$-dimensional simplex with edges of length $\sqrt{2}$. Then the volume of any $k$-dimensional slice of $S$, passing through the centroid, is at most $\sqrt{\frac{n+1}{n-k+1}} \frac{1}{k !}$. This upper bound is attained if and only if the slice contains $k$ vertices of $S$.

We will show that this result would follow from the conjecture below, which concerns the position of the centroid of central slices of a regular $(n-1)$-simplex. Conjecture 2.2 Let $S$ be a regular(n-1)-dimensional simplex and $T$ a $k$-dimensional slice of $S$ containing the centre of mass. Then the distance from the centroid of $T$ to the centroid of $S$ is maximised when $T$ contains $k$ vertices of $S$.

The codimension of the slices is reduced by 1 between Conjecture 2.1 and Conjecture 2.2. This means that we have another proof of the 1-codimensional case because when $k=n-1$ Conjecture 2.2 is trivial. 
We attempt to prove Conjecture 2.1 by combining the methods of Chapter 1 with induction on the codimension of the slice. The result is trivial for $n-k=0$ ( and in Chapter 1 we proved that the result holds when $n-k=1$ ).

Suppose then that $1 \leq k \leq n-1$ and that $H$ is a $(k+1)$-dimensional subspace of $R^{n+1}$ that contains the point $(1, \ldots, 1)$. If we take

$$
S_{t}=\left\{\mathrm{x}=\left(x_{1}, \ldots, x_{n+1}\right) \in R^{n+1}: x_{i} \geq 0 \quad i=1, \ldots, n+1 \quad, \quad \sum_{i=1}^{n+1} x_{i}=t\right\}
$$

for each $t>0$, then $H \cap S_{t}$ is a $k$-dimensional central slice of the regular $n$-simplex $S_{t}$. As in Chapter 1 we will estimate $\operatorname{Vol}_{k}\left(H \cap S_{1}\right)$.

Define functions $f: R \rightarrow[0, \infty)$ and $F: R^{n+1} \rightarrow[0, \infty)$ by

$$
\begin{gathered}
f(x)= \begin{cases}e^{-x} & \text { if } x \geq 0 \\
0 & \text { otherwise }\end{cases} \\
F(\mathbf{x})=\prod_{j=1}^{n+1} f\left(<\mathbf{x}, \mathbf{e}_{j}>\right)
\end{gathered}
$$

For a fixed $t>0, F$ is constant on $S_{t}$ and

$$
\operatorname{Vol}_{k}\left(H \bigcap S_{t}\right)=t^{k} \operatorname{Vol}_{k}\left(H \bigcap S_{1}\right)
$$

so a change of coordinates gives

$$
\begin{aligned}
\int_{H} F d V o l_{H} & =\int_{H} \prod_{j=1}^{n+1} f\left(x_{j}\right) d V o l_{H} \\
& =\int_{0}^{\infty} e^{-s \sqrt{n+1}} \operatorname{Vol}_{k}\left(H \bigcap S_{s \sqrt{n+1}}\right) d s \\
& =\int_{0}^{\infty} e^{-s \sqrt{n+1}}(s \sqrt{n+1})^{k} \operatorname{Vol}_{k}\left(H \bigcap S_{1}\right) d s \\
& =k !(n+1)^{-\frac{1}{2}} \operatorname{Vol}_{k}\left(H \bigcap S_{1}\right)
\end{aligned}
$$


that is

$$
\operatorname{Vol}_{k}\left(H \bigcap S_{1}\right)=\frac{\sqrt{n+1}}{k !} \int_{H} F d V o l_{H}
$$

Our problem is to show that for any $(k+1)$-dimensional subspace $H$ in $R^{n+1}$, containing $(1, \ldots, 1)$,

$$
\int_{H} F d V o l_{H} \leq \frac{1}{\sqrt{n-k+1}}
$$

We divide the proof into two parts. The first part generalises the use of Hölders inequality in Theorem 1.4. The second part uses the fact that $F$ is a log concave function, and it is this part that we reduce to Conjecture 2.2 .

We begin by stating a result of Brascamp and Lieb [6] concerning the estimation of convolutions.

Theorem 2.3 (Brascamp and Lieb) Let $u_{1}, \ldots, u_{m}$ be a sequence of unit vectors in $R^{n}, m \geq n$, and $c_{1}, \ldots, c_{m}$ a sequence of positive real numbers satisfying

$$
\sum_{i=1}^{m} c_{i} u_{i} \otimes u_{i}=I_{n}
$$

where $I_{n}$ denotes the identity on $R^{n}$. Then for integrable functions $f_{1}, \ldots, f_{m}: R^{n} \rightarrow[0, \infty)$,

$$
\int_{R^{n}} \prod_{i=1}^{m} f_{i}\left(<u_{i}, x>\right)^{c_{i}} d x \leq \prod_{i=1}^{m}\left(\int_{R} f_{i}\right)^{c_{i}}
$$

There is equality if the $f_{i}$ are identical Gaussian densities or if the $u_{i}$ for an orthonormal basis of $R^{n}$.

Let $P$ denote the orthogonal projection of $R^{n+1}$ onto $H$ and define

$$
c_{j}=\left\|P \mathbf{e}_{j}\right\|^{2}
$$




$$
\mathbf{u}_{j}=\frac{P \mathbf{e}_{j}}{\sqrt{c_{j}}}
$$

for each $j, 1 \leq j \leq n+1$.

Then

$$
\sum_{i=1}^{n+1} c_{i} \mathbf{u}_{i} \otimes \mathbf{u}_{i}=\sum_{i=1}^{n+1} P \mathbf{e}_{i} \otimes P \mathbf{e}_{i}=I_{H}
$$

where $I_{H}$ denotes the identity on the subspace $H$.

By the equality of the traces of these operators we have,

$$
\sum_{i=1}^{n+1} c_{j}=k+1
$$

If $\mathbf{x} \in H$ then for each $1 \leq j \leq n+1$,

$$
<\mathbf{x}, \mathbf{e}_{j}>=<\mathbf{x}, P \mathbf{e}_{j}>
$$

so that,

$$
\begin{aligned}
\int_{H} \prod_{j=1}^{n+1} f\left(<\mathbf{x}, \mathbf{e}_{j}>\right) d V o l_{H} & =\int_{H} \prod_{j=1}^{n+1} f\left(<\mathbf{x}, P \mathbf{e}_{j}>\right) d V o l_{H} \\
& =\int_{H} \prod_{j=1}^{n+1} f\left(<\sqrt{c_{j}} \mathbf{x}, \mathbf{u}_{j}>\right) d V o l_{H}
\end{aligned}
$$

Applying the Brascamp-Lieb inequality,

$$
\begin{aligned}
\int_{H} F d V o l_{H} & \leq \prod_{j=1}^{n+1}\left(\int_{R} f\left(\sqrt{c_{j}} x\right)^{\frac{1}{c_{j}}} d x\right)^{c_{j}} \\
& =\prod_{j=1}^{n+1}\left(\int_{0}^{\infty} e^{\frac{-\pi}{\sqrt{c_{j}}}} d x\right)^{c_{j}} \\
& =\prod_{j=1}^{n+1}\left(c_{j}^{c_{j}}\right)^{\frac{1}{2}}
\end{aligned}
$$


Since $S_{1}$ spans a hyperplane $H^{*}$ which is perpendicular to the vector $(1, \ldots, 1)$ and $H$ contains the line $\{(\alpha, \ldots, \alpha): \alpha \in R\}$, the projection of any point of $S_{1}$ onto $H$ lies in $H^{*}$. Therefore, for each $1 \leq j \leq n+1$,

$$
c_{j}=\left\|P \mathbf{e}_{j}\right\|^{2} \geq \frac{1}{n+1}
$$

We note in passing that this fact is enough to show that the central slices of $S_{1}$ are strictly smaller than the $k$-dimensional faces since

$$
\begin{aligned}
\int_{H} F d V o l_{H} & \leq \prod_{j=1}^{n+1}\left(c_{j}^{c_{j}}\right)^{\frac{1}{2}} \\
& <\prod_{j=1}^{n+1}\left(c_{j}^{\frac{1}{n+1}}\right)^{\frac{1}{2}}
\end{aligned}
$$

and, using the AM/GM inequality,

$$
\begin{aligned}
\int_{H} F d V o l_{H} & <\sqrt{\frac{1}{n+1} \sum_{i=1}^{n+1} c_{i}} \\
& =\sqrt{\frac{k+1}{n+1}}
\end{aligned}
$$

That is,

$$
\operatorname{Vol}_{k}\left(H \bigcap S_{1}\right)<\frac{\sqrt{k+1}}{k !}
$$

which is the volume of a $k$-dimensional face of $S_{1}$.

However, our conjecture is the stronger inequality,

$$
\int_{H} F d V o l_{H} \leq \frac{1}{\sqrt{n-k+1}}
$$

We break the proof into two parts, the first for when for every $1 \leq j \leq n+1$ we have $\frac{1}{n-k+1} \leq c_{j} \leq 1$, and the second for when $c_{j}<\frac{1}{n-k+1}$ for some $j$. 
The break at $c_{j}=\frac{1}{n-k+1}$ corresponds to the break in the proof in Chapter 1 at $a_{j}=\sqrt{\frac{1}{2}}$ because when $k=n-1$ we have $c_{j}=1-a_{j}^{2}$ for each $1 \leq j \leq n+1$.

CASE 1. $\frac{1}{n-k+1} \leq c_{j} \leq 1$ for each $j=1, \ldots, n+1$.

We have,

$$
0<\frac{1}{n+1} \sum_{i=1}^{n+1} c_{j}=\frac{k+1}{n+1}<1
$$

so by the convexity of the function $f(t)=t^{t}$ on $[0,1]$ we see that the product

$$
\prod_{j=1}^{n+1} c_{j}^{c_{j}}
$$

is maximised when $k$ of the $c_{j}$ are equal to 1 and the remaining $n-k+1$ are equal to $\frac{1}{n-k+1}$. That is,

$$
\prod_{j=1}^{n+1} c_{j}^{c_{j}} \leq \frac{1}{n-k+1}
$$

so that

$$
\int_{H} F d V o l_{H} \leq \frac{1}{\sqrt{n-k+1}}
$$

Equality occurs in this case only if $k$ of the $c_{j}$ are equal to 1 . That is, $P \mathbf{e}_{j}=\mathbf{e}_{j}$ for $k$ of the standard basis vectors, meaning the slice contains $k$ vertices of $S_{1}$.

CASE 2. $\frac{1}{n+1} \leq c_{j}<\frac{1}{n-k+1}$ for some $1 \leq j \leq n+1$.

Without loss of generality we may assume that

$$
\frac{1}{n+1} \leq c_{1}<\frac{1}{n-k+1}
$$

This corresponds to the difficult $a_{1}>\frac{1}{\sqrt{2}}$ case in Chapter 1 . We will show that this case will reduce to Conjecture 2.2 . 
Define functions $G: R^{n+1} \rightarrow[0, \infty)$ and $g: R \rightarrow[0, \infty)$ by,

$$
\begin{gathered}
G(\mathbf{x})=\prod_{j=2}^{n+1} f\left(<\mathbf{x}, \mathbf{e}_{j}>\right) \\
g(t)=\int_{H \bigcap\left\{\mathbf{x}:<\mathbf{x}, \mathbf{e}_{1}>=t\right\}} G(\mathbf{x}) d \lambda
\end{gathered}
$$

where $\lambda$ denotes the Lebesgue measure on the set $H \bigcap\left\{\mathbf{x}:<\mathbf{x}, \mathbf{e}_{1}>=t\right\}$.

By changing variables we may write the following integrals over $H$ as integrals over $[0, \infty)$.

$$
\begin{aligned}
\int_{H} F d V o l_{H} & =\frac{1}{\sqrt{c_{1}}} \int_{0}^{\infty} e^{-t} g(t) d t \\
\int_{H} G d V o l_{H} & =\frac{1}{\sqrt{c_{1}}} \int_{0}^{\infty} g(t) d t \\
\int_{H} G(\mathbf{x})<\mathbf{x}, \mathbf{e}_{1}>d V o l_{H} & =\frac{1}{\sqrt{c_{1}}} \int_{0}^{\infty} t g(t) d t
\end{aligned}
$$

Since $g$ is a positive $\log$ concave function on $R$ we may apply Lemma 1.3 from Chapter 1 and obtain,

$$
\int_{0}^{\infty} e^{-t} g(t) d t \leq \frac{\left(\int_{0}^{\infty} g(t) d t\right)^{2}}{\int_{0}^{\infty} g(t)(t+1) d t}
$$

That is,

$$
\int_{H} F d V o l_{H} \leq \frac{\left(\int_{H} G d V o l_{H}\right)^{2}}{\int_{H} G(\mathbf{x})\left(<\mathbf{x}, \mathbf{e}_{1}>+1\right) d V o l_{H}}
$$

Let $P_{1}$ denote the orthogonal projection of $R^{n+1}$ onto the subspace perpendicular to $\mathbf{e}_{1}$.

As $G$ does not involve the first co-ordinate of points in $R^{n+1}$, the integral of $G$ over $H$ is a simple multiple of the integral of $G$ over $P_{1} H$,

$$
\int_{H} G d V o l_{H}=\frac{1}{\sqrt{1-c_{1}}} \int_{P_{1} H} G d V o l_{P_{1} H}
$$


However, $P_{1} H$ is a $(k+1)$-dimensional subspace of $R^{n}=\mathbf{e}_{1}^{\perp}$ containing the point $(1, \ldots, 1)$, so we may interpret this last integral in terms of the volume of a $k$ dimensional central section of an $(n-1)$-dimensional simplex. That is we may interpret the integral in terms of an $(n-k-1)$-codimensional central section and so apply our inductive assumption. Therefore,

$$
\int_{P_{1} H} G d V o l_{P_{1} H} \leq \frac{1}{\sqrt{n-k}}
$$

and,

$$
\int_{H} G d V o l_{H} \leq \frac{1}{\sqrt{1-c_{1}}} \frac{1}{\sqrt{n-k}}
$$

To complete the proof it would be sufficient to show that

$$
\int_{H} G(\mathbf{x})<\mathbf{x}, \mathbf{e}_{1}>d V o l_{H} \geq \frac{1}{n-k} \int_{H} G d V o l_{H}
$$

because then we would have, from (2.1),

$$
\begin{aligned}
\int_{H} F d V o l_{H} & \leq \frac{\left(\int_{H} G d V o l_{H}\right)^{2}}{\int_{H} G(\mathbf{x})\left(<\mathbf{x}, \mathbf{e}_{1}>+1\right) d V o l_{H}} \\
& \leq \frac{\left(\int_{H} G d V o l_{H}\right)^{2}}{\left(1+\frac{1}{n-k}\right) \int_{H} G d V o l_{H}} \\
& =\frac{n-k}{n-k+1} \int_{H} G d V o l_{H}
\end{aligned}
$$

so, by the inductive assumption,

$$
\begin{aligned}
\int_{H} F d V o l_{H} & \leq \frac{n-k}{n-k+1} \quad \frac{1}{\sqrt{1-c_{1}}} \frac{1}{\sqrt{n-k}} \\
& =\frac{\sqrt{n-k}}{n-k+1} \frac{1}{\sqrt{1-c_{1}}}
\end{aligned}
$$


and since $c_{1}<\frac{1}{n-k+1}$,

$$
\int_{H} F d \operatorname{Vol}_{H}<\frac{1}{n-k+1}
$$

as required.

Therefore the problem has been reduced to showing

$$
\int_{H} G(\mathbf{x})<\mathbf{x}, \mathbf{e}_{1}>d V o l_{H} \geq \frac{1}{n-k} \int_{H} G d V o l_{H}
$$

whenever $c_{1}<\frac{1}{n-k+1}$.

Writing $P \mathbf{e}_{1}=\left(v_{1}, \ldots, v_{n+1}\right)$ we have,

$$
\begin{aligned}
v_{1} & =c_{1} \\
\sum_{i=1}^{n+1} v_{i} & =1 \\
\sum_{i=1}^{n+1} v_{i}^{2} & =c_{1}
\end{aligned}
$$

and

$$
\begin{aligned}
\int_{H} G(\mathbf{x})<\mathbf{x}, \mathbf{e}_{1}>d V o l_{H} & =\int_{H} G(\mathbf{x})<\mathbf{x}, P \mathbf{e}_{1}>d V o l_{H} \\
& =\sum_{j=1}^{n+1} v_{j} \int_{H} G(\mathbf{x})<\mathbf{x}, \mathbf{e}_{j}>d V o l_{H}
\end{aligned}
$$

Therefore,

$$
\left(1-v_{1}\right) \int_{H} G(\mathbf{x})<\mathbf{x}, \mathbf{e}_{1}>d V o l_{H}=\sum_{j=2}^{n+1} v_{j} \int_{H} G(\mathbf{x})<\mathbf{x}, \mathbf{e}_{j}>d V o l_{H}
$$

so that,

$$
\int_{H} G(\mathbf{x})<\mathbf{x}, \mathbf{e}_{1}>d V o l_{H}=\frac{1}{1-c_{1}} \sum_{j=2}^{n+1} v_{j} \int_{H} G(\mathbf{x})<\mathbf{x}, \mathbf{e}_{j}>d V o l_{H}
$$




$$
=\left(\frac{1}{1-c_{1}}\right)^{\frac{3}{2}} \sum_{j=2}^{n+1} v_{j} \int_{P_{1} H} G(\mathbf{y})<\mathbf{y}, \mathbf{e}_{j}>d \operatorname{Vol}_{P_{1} H}
$$

When $k=n-1$ the problem is now trivial, since $P_{1} H=R^{n}$ and so, for each $2 \leq j \leq n+1$

$$
\begin{aligned}
\int_{P_{1} H} G(\mathbf{y})<\mathbf{y}, \mathbf{e}_{j}>d V o l_{P_{1} H} & =1 \\
& =\int_{P_{1} H} G(\mathbf{y}) d V o l_{P_{1} H}
\end{aligned}
$$

Hence

$$
\begin{aligned}
\int_{H} G(\mathbf{x})<\mathbf{x}, \mathbf{e}_{1}>d V o l_{H} & =\left(\frac{1}{1-c_{1}}\right)^{\frac{3}{2}} \sum_{j=2}^{n+1} v_{j} \int_{P_{1} H} G(\mathbf{y})<\mathbf{y}, \mathbf{e}_{j}>d V o l_{P_{1} H} \\
& =\left(\frac{1}{1-c_{1}}\right)^{\frac{3}{2}} \sum_{j=2}^{n+1} v_{j} \int_{P_{1} H} G(\mathbf{y}) d V o l_{P_{1} H} \\
& =\frac{1}{1-c_{1}} \sum_{j=2}^{n+1} v_{j} \int_{H} G(\mathbf{x}) d V o l_{H} \\
& =\int_{H} G(\mathbf{x}) d V o l_{H}
\end{aligned}
$$

When $k<n-1$ the problem is not so straightforward, but may be reduced to Conjecture 2.2 .

We wish to show that

$$
\int_{H} G(\mathbf{x})<\mathbf{x}, \mathbf{e}_{1}>d V o l_{H} \geq \frac{1}{n-k} \int_{H} G d V o l_{H}
$$

Writing

$$
A_{j}=\int_{P_{1} H} G(\mathbf{y})<\mathbf{y}, \mathbf{e}_{j}>d V o l_{P_{1} H}
$$


for each $2 \leq j \leq n+1$, we may write (2.2) as,

$$
\int_{H} G(\mathbf{x})<\mathbf{x}, \mathbf{e}_{1}>d V o l_{H}=\left(\frac{1}{1-c_{1}}\right)^{\frac{3}{2}} \sum_{i=2}^{n+1} v_{i} A_{i}
$$

where,

$$
\begin{aligned}
& \sum_{i=2}^{n+1} v_{i}=1-c_{1} \\
& \sum_{i=2}^{n+1} v_{i}^{2}=c_{1}\left(1-c_{1}\right)
\end{aligned}
$$

Also, using the same change of coordinates as earlier,

$$
\begin{aligned}
\sum_{i=2}^{n+1} A_{i} & =\int_{P_{1} H} G(\mathbf{y}) \sum_{i=2}^{n+1}<\mathbf{y}, \mathbf{e}_{i}>d \operatorname{Vol}_{P_{1} H} \\
& =\int_{P_{1} H} \prod_{i=2}^{n+1} f\left(<\mathbf{y}, \mathbf{e}_{i}>\right)\left(\sum_{i=2}^{n+1}<\mathbf{y}, \mathbf{e}_{i}>\right) d \operatorname{Vol}_{P_{1} H} \\
& =\int_{0}^{\infty} e^{-s \sqrt{n}}(s \sqrt{n}) \operatorname{Vol}_{k}\left(H \bigcap S_{s \sqrt{n}}\right) d s \\
& =\int_{0}^{\infty} e^{-s \sqrt{n}}(s \sqrt{n})^{k+1} \operatorname{Vol}_{k}\left(H \bigcap S_{1}\right) d s \\
& =(k+1) \int_{0}^{\infty} e^{-s \sqrt{n}}(s \sqrt{n})^{k} \operatorname{Vol}_{k}\left(H \bigcap S_{1}\right) d s \\
& =(k+1) \int_{P_{1} H} G(\mathbf{y}) d \operatorname{Vol}_{P_{1} H}
\end{aligned}
$$

Writing

$$
A=\int_{P_{1} H} G(\mathbf{y}) d V o l_{P_{1} H}
$$

we have,

$$
\sum_{i=2}^{n+1} A_{i}=(k+1) A
$$

We now minimise

$$
\sum_{i=2}^{n+1} v_{i} A_{i}
$$


subject to the conditions,

$$
\begin{aligned}
& \sum_{\substack{i=2 \\
n+1}}^{n+1} v_{i}=1-c_{1} \\
& \sum_{i=2}^{n+1} v_{i}^{2}=c_{1}\left(1-c_{1}\right)
\end{aligned}
$$

using Lagrange multipliers.

After differentiating with respect to each $v_{i}, 2 \leq i \leq n+1$, we have that the $A_{i}$ and $v_{i}$ must satisfy,

$$
A_{i}+\mu+2 \lambda v_{i}=0
$$

for each $2 \leq i \leq n+1$, where $\lambda, \mu \in R$.

Summing (2.4) over $2 \leq i \leq n+1$ and rearranging, we have

$$
\mu=-\frac{1}{n}\left(\sum_{i=2}^{n+1} A_{i}+2 \lambda\left(1-c_{1}\right)\right)
$$

so $(2.4)$ can be rewritten,

$$
A_{i}-\frac{1}{n} \sum_{i=2}^{n+1} A_{i}=2 \lambda\left(\frac{1}{n}\left(1-c_{1}\right)-v_{i}\right)
$$

for each $2 \leq i \leq n+1$.

Squaring each side of (2.5) and summing over $2 \leq i \leq n+1$,

$$
n \sum_{i=2}^{n+1} A_{i}^{2}-\left(\sum_{i=2}^{n+1} A_{i}\right)^{2}=\left((n+1) c_{1}-1\right)\left(1-c_{1}\right) 4 \lambda^{2}
$$

so that,

$$
\lambda= \pm \sqrt{\frac{n \sum_{i=2}^{n+1} A_{i}^{2}-\left(\sum_{i=2}^{n+1} A_{i}\right)^{2}}{\left((n+1) c_{1}-1\right)\left(1-c_{1}\right)}}
$$


Therefore from (2.5),

$$
A_{i}-\frac{1}{n} \sum_{i=2}^{n+1} A_{i}= \pm \sqrt{\frac{n \sum_{i=2}^{n+1} A_{i}^{2}-\left(\sum_{i=2}^{n+1} A_{i}\right)^{2}}{\left((n+1) c_{1}-1\right)\left(1-c_{1}\right)}}\left(v_{i}-\frac{1}{n}\left(1-c_{1}\right)\right)
$$

for each $2 \leq i \leq n+1$.

Multiplying (2.6) by $v_{i}$ and summing over $2 \leq i \leq n+1$,

$$
\sum_{i=2}^{n+1} v_{i} A_{i}=\frac{1-c_{1}}{n}\left(\sum_{i=2}^{n+1} A_{i} \pm \sqrt{n \sum_{i=2}^{n+1} A_{i}^{2}-\left(\sum_{i=2}^{n+1} A_{i}\right)^{2}} \sqrt{\frac{n c_{1}}{1-c_{1}}-1}\right)
$$

so the minimum occurs when

$$
\sum_{i=2}^{n+1} v_{i} A_{i}=\frac{1-c_{1}}{n}\left(\sum_{i=2}^{n+1} A_{i}-\sqrt{n \sum_{i=2}^{n+1} A_{i}^{2}-\left(\sum_{i=2}^{n+1} A_{i}\right)^{2}} \sqrt{\frac{n c_{1}}{1-c_{1}}-1}\right)
$$

Using

$$
\sum_{i=2}^{n+1} A_{i}=(k+1) A
$$

and,

$$
\frac{c_{1}}{1-c_{1}} \leq \frac{1}{n-k}
$$

we have

$$
\int_{H} G(\mathbf{x})<\mathbf{x}, \mathbf{e}_{1}>d V o l_{H} \geq \frac{1}{n \sqrt{1-c_{1}}}\left((k+1) A-\sqrt{n \sum_{i=2}^{n+1} A_{i}^{2}-\left(\sum_{i=2}^{n+1} A_{i}\right)^{2}} \sqrt{\frac{k}{n-k}}\right)
$$

If we could show that

$$
\sum_{i=2}^{n+1} A_{i}^{2} \leq\left(k+\frac{1}{n-k}\right) A^{2}
$$


then from (2.7) we would have the required result, since,

$$
\begin{aligned}
\int_{H} G(\mathbf{x})<\mathbf{x}, \mathbf{e}_{1}>d V o l_{H} & \geq \frac{1}{(n-k) \sqrt{1-c_{1}}} A \\
& =\frac{1}{n-k} \int_{H} G(\mathbf{x}) d V o l_{H}
\end{aligned}
$$

Inequality (2.8) may be written as the following conjecture :

Conjecture 2.4 Let $K$ be a $(k+1)$-dimensional subspace of $R^{n}$ containing the point $(1, \ldots, 1)$, and let $f: R \rightarrow R$ and $G: R^{n} \rightarrow R$ be defined by

$$
\begin{gathered}
f(x)= \begin{cases}e^{-x} & \text { if } x \geq 0 \\
0 & \text { otherwise }\end{cases} \\
G(\mathbf{x})=\prod_{j=1}^{n} f\left(<\mathbf{x}, \mathbf{e}_{j}>\right)
\end{gathered}
$$

Then

$$
\sum_{j=1}^{n}\left(\int_{K} G(\mathbf{y})<\mathbf{y}, \mathbf{e}_{j}>d V o l_{K}\right)^{2} \leq\left(k+\frac{1}{n-k}\right)\left(\int_{K} G(\mathbf{y}) d V o l_{K}\right)^{2}
$$

This may be reinterpreted as a conjecture about the centroid of central slices of a regular $(n-1)$-dimensional simplex since, for each $1 \leq i \leq n$,

$$
\begin{aligned}
\int_{K} G(\mathbf{y})<\mathbf{y}, \mathbf{e}_{i}>d V o l_{K} & =\frac{1}{\sqrt{n}} \int_{0}^{\infty} e^{-t}\left(\int_{K \bigcap s_{t}}<\mathbf{y}, \mathbf{e}_{i}>d V o l_{K \cap s_{t}}\right) d t \\
& =\frac{1}{\sqrt{n}} \int_{0}^{\infty} e^{-t} t \bar{y}_{i} \operatorname{Vol}_{k}\left(K \bigcap S_{t}\right) d t
\end{aligned}
$$

where $\bar{y}_{i}$ denotes the $i$ th coordinate of $\overline{\mathbf{y}}$, the centroid of $K \cap S_{1}$. Therefore,

$$
\int_{K} G(\mathbf{y})<\mathbf{y}, \mathbf{e}_{i}>d V o l_{K}=\bar{y}_{i}(k+1) \int_{K} G(\mathbf{y}) d V o l_{K}
$$


for each $1 \leq i \leq n$, and the conjecture is,

$$
\sum_{i=1}^{n} \bar{y}_{i}^{2} \leq \frac{1}{(k+1)^{2}}\left(k+\frac{1}{n-k}\right)
$$

Equality is achieved if and only if $K$ contains $k$ vertices of $S_{1}$, so the conjecture is exactly Conjecture 2.2.

Again, when $k=n-1$ the result is trivial because $K \cap S_{1}$ is an $(n-1)$ dimensional central slice of an $(n-1)$-dimensional simplex and so the centroid of the slice is

$$
\overline{\mathbf{y}}=\left(\frac{1}{n}, \ldots, \frac{1}{n}\right)
$$

giving,

$$
\sum_{i=1}^{n} \bar{y}_{i}^{2}=\frac{1}{n}
$$




\section{Chapter 3}

In this chapter we will examine maximal ellipsoids in central slices of the regular $n$-simplex. We will derive a complete solution to the maximal slice problem in the cases $k=1$ and $k=2$. We noted in the introduction that the $k=1$ case is very easy but we include a slightly different proof here as the method provides an insight into the main result which states

Proposition 3.1 Over all 2-dimensional ellipsoids lying in the central sections of a regular $n$-simplex, $n \geq 4$, the ones with largest volume are the maximal ellipses of the triangular sections of the simplex containing two of the vertices.

Although this does not cover the simplex in 3 dimensions, by combining Proposition 3.1 with the volume ratio result in [4], and Theorem 1.4 we have, Corollary 3.1 The 2-dimensional slices of a regular $n$-simplex through its centroid with largest volume are those slices containing two of the vertices.

We prove Proposition 3.1 by reducing it to a question about determinants of $2 \times 2$ matrices of the form $\sum_{i=1}^{n+1} z_{i} \otimes z_{i}$ where $\left(z_{i}\right)_{i=1}^{n+1}$ is a sequence of vectors in $R^{2}$ satisfying certain conditions. To estimate these determinants we firstly make estimates 
on the length of $z_{i} \wedge z_{j}$ for pairs of vectors $i \neq j$, and then sum the expressions over all pairs $1 \leq i<j \leq n+1$. Determinants of this type occur in various areas of mathematics, so we hope that the techniques used here may be applicable to other problems.

\section{The Setup}

Our aim in this section is to reduce the problem of calculating volumes of $k$ dimensional ellipsoids in $R^{n}$ to that of calculating the determinant of a $k \times k$ matrix. It will be shown that the precise problem is to determine the least $E(n, k)$ such that

$$
\operatorname{Det}\left(\sum_{i=1}^{n+1} z_{i} \otimes z_{i}\right) \leq\left(\frac{n+1}{n}\right)^{k} E(n, k)^{2}
$$

for every sequence $\left(z_{i}\right)_{i=1}^{n+1}$ in $R^{k}$ which satisfies

$$
\sum_{i=1}^{n+1} z_{i}=0
$$

and

$$
<z_{i}, x>+\left\|z_{i}\right\| \leq 1 \quad i=1, \ldots, n+1
$$

for some $x \in R^{k}$.

Let $S$ be the regular simplex in $R^{n}$ :

$$
S=\left\{x \in R^{n}: \quad<x, u_{i}>\leq 1 \quad i=1, \ldots, n+1\right\}
$$

where $\left(u_{i}\right)_{i=1}^{n+1}$ is a sequence of unit vectors satisfying

$$
\sum_{i=1}^{n+1} u_{i}=0
$$


and

$$
\frac{n}{n+1} \sum_{i=1}^{n+1} u_{i} \otimes u_{i}=I_{n}
$$

where $I_{n}$ denotes the identity on $R^{n}$.

Let

$$
\varepsilon=\left\{x \in R^{n}: \sum_{j=1}^{k} \alpha_{j}^{-2}<x-y, v_{j}>^{2} \leq 1, \quad<x, v_{j}>=0, \quad k+1 \leq j \leq n\right\}
$$

be a $k$-dimensional ellipsoid, where $\left(v_{j}\right)_{j=1}^{n}$ is an orthonormal basis of $R^{n}, y \in R^{n}$ and $\left(\alpha_{j}\right)_{j=1}^{k}$ is a sequence of positive real numbers.

We assume that $\varepsilon$ lies in a slice of $S$ containing the origin. This is exactly the statement that $y \in \operatorname{span}\left(v_{j}\right)_{j=1}^{k}$. The problem is to find a best upper bound, $E(n, k)$, for the product $\prod_{j=1}^{k} \alpha_{j}$.

For each $i, 1 \leq i \leq n+1$ the point

$$
x_{i}=y+\frac{\sum_{j=1}^{k} \alpha_{j}^{2}<u_{i}, v_{j}>v_{j}}{\left(\sum_{j=1}^{k} \alpha_{j}^{2}<u_{i}, v_{j}>^{2}\right)^{\frac{1}{2}}}
$$

lies in $\varepsilon$. Since $\varepsilon \subset S$ this means that for each $i$,

$$
<u_{i}, y>+\left(\sum_{j=1}^{k} \alpha_{j}^{2}<u_{i}, v_{j}>^{2}\right)^{\frac{1}{2}} \leq 1
$$

Define the map $T: R^{n} \rightarrow R^{n}$ by

$$
T z=\sum_{j=1}^{k} \alpha_{j}<z, v_{j}>v_{j}
$$

Then for every $i, 1 \leq i \leq n+1$,

$$
<u_{i}, y>+\left\|T u_{i}\right\| \leq 1
$$


Let $P$ denote the orthogonal projection of $R^{n}$ onto $\operatorname{span}\left(v_{j}\right)_{j=1}^{k}$ and write $w_{i}=P u_{i}$. For each $i$,

$$
T u_{i}=T w_{i}
$$

and

$$
<u_{i}, y>=<w_{i}, y>
$$

so the restriction of $T$ to $\operatorname{span}\left(v_{j}\right)_{j=1}^{k}$ is an isomorphism and our problem is to estimate $\operatorname{Det}(T)$.

Rewriting everything in terms of the $w_{i}$ and using $T$ to denote the restriction $\left.T\right|_{s p a n\left(v_{j}\right)_{1}^{k}}$ we have a sequence $\left(w_{i}\right)_{i=1}^{n+1}$ of vectors in $R^{k}$ satisfying

$$
\sum_{i=1}^{n+1} w_{i}=0
$$

and

$$
\frac{n}{n+1} \sum_{i=1}^{n+1} w_{i} \otimes w_{i}=I_{k}
$$

a vector $y \in R^{k}$ and a linear map $T$ such that for each $i, 1 \leq i \leq n+1$,

$$
<w_{i}, y>+\left\|T w_{i}\right\| \leq 1
$$

and we wish to estimate $\operatorname{Det}(T)$.

The problem may be reformulated slightly by writing $z_{i}=T w_{i}$ for each $i$ and $x=T^{-1} y$. Then

$$
T^{2}=\frac{n}{n+1} \sum_{i=1}^{n+1} z_{i} \otimes z_{i}
$$

and by the self-adjointness of $T$,

$$
<w_{i}, y>=<w_{i}, T x>=<T w_{i}, x>=<z_{i}, x>
$$


for each $i$. The problem is then reduced to finding an upper bound $E(n, k)$ such that

$$
\operatorname{Det}\left(\sum_{i=1}^{n+1} z_{i} \otimes z_{i}\right) \leq\left(\frac{n+1}{n}\right)^{k} E(n, k)^{2}
$$

for every sequence $\left(z_{i}\right)_{i=1}^{n+1}$ in $R^{k}$ which satisfies the following two conditions,

$$
\begin{aligned}
& \text { (A) } \quad<z_{i}, x>+\left\|z_{i}\right\| \leq 1 \quad 1 \leq i \leq n+1 \\
& \text { (B) } \quad \sum_{i=1}^{n+1} z_{i}=0
\end{aligned}
$$

for some $x \in R^{k}$.

\section{Calculating $E(n, k)$}

We now give values for the best upper bound $E(n, k)$ when $k=1$ and $k=2$.

As mentioned earlier, the $k=1$ case is much simpler since it only involves the calculation of the length of a straight line in $S$ passing through the centroid, and could be done more directly. However, the technique used here is similar to that required in the more difficult result for $k=2$.

Proposition 3.2 Under the conditions given above, for $n \geq 1, E(n, 1)=\frac{n+1}{2}$. This bound is attained when the 1-dimensional slice of $S$ passes through a vertex.

Proof When $k=1$ the conditions above are,
(A)

$$
\theta z_{i}+\left|z_{i}\right| \leq 1
$$$$
1 \leq i \leq n+1
$$$$
\text { (B) } \quad \sum_{i=1}^{n+1} z_{i}=0
$$ 
for a sequence $\left(z_{i}\right)_{i=1}^{n+1}$ in $R, \theta \in R$, and we show

$$
\sum_{i=1}^{n+1} z_{i}^{2} \leq \frac{(n+1)^{3}}{4 n}
$$

Without loss of generality we may take $\theta \geq 0$ and split the proof into two cases, $\theta \leq \frac{n-1}{n+1}$ and $\theta \geq \frac{n-1}{n+1}$.

CASE 1. $\theta \leq \frac{n-1}{n+1}$

By condition $(A)$,

$$
\begin{aligned}
\sum_{i=1}^{n+1} z_{i}^{2} & \leq \sum_{i=1}^{n+1}\left(1-\theta z_{i}\right)^{2} \\
& =(n+1)-2 \theta \sum_{i=1}^{n+1} z_{i}+\theta^{2} \sum_{i=1}^{n+1} z_{i}^{2} \\
& =(n+1)+\theta^{2} \sum_{i=1}^{n+1} z_{i}^{2}
\end{aligned}
$$

by condition $(B)$.

Therefore,

$$
\begin{aligned}
\sum_{i=1}^{n+1} z_{i}^{2} & \leq \frac{n+1}{1-\theta^{2}} \\
& \leq \frac{(n+1)^{3}}{4 n}
\end{aligned}
$$

CASE 2. $\theta \geq \frac{n-1}{n+1}$

By condition $(A)$, for each $i, 1 \leq i \leq n+1$

$$
z_{i} \leq \frac{1}{1+\theta}
$$

and so by condition $(B)$ we also have for each $i$,

$$
z_{i} \geq-\frac{n}{1+\theta}
$$


Therefore,

$$
\sum_{i=1}^{n+1}\left(z_{i}-\frac{1}{1+\theta}\right)\left(z_{i}+\frac{n}{1+\theta}\right) \leq 0
$$

and so, after expanding this expression and using condition $(B)$,

$$
\begin{aligned}
\sum_{i=1}^{n+1} z_{i}^{2} & \leq \frac{n(n+1)}{(1+\theta)^{2}} \\
& \leq \frac{(n+1)^{3}}{4 n}
\end{aligned}
$$

In the extreme case $\theta=\frac{n-1}{n+1}$, with $n$ of the $z_{i}$ equal to $\frac{n+1}{2 n}$ and remaining $z_{i}$ equal to $\frac{-n(n+1)}{2 n}$, that is, all but one of the $u_{i}$ project to the same point of $\operatorname{span}\left(v_{1}\right)$, so the line must lie perpendicular to a facet of $S$.

The approach to the 2-dimensional case is similar. The proof again splits into two cases at $\theta=\frac{n-1}{n+1}$. In the case $\theta \geq \frac{n-1}{n+1}$ we use the fact that the determinant may be written as a sum over pairs of vectors. Again we use inequalities obtained by summing products of positive expressions, but the difference is that we now sum over pairs $1 \leq i<j \leq n+1$ instead of summing over $1 \leq i \leq n+1$.In the case $\theta \leq \frac{n-1}{n+1}$ when $n \geq 5$ we can use an easy trace estimate for the determinant. This estimate is not sufficient when $n<5$ but can be combined with an argument similar to the first case to complete the proof for $n=4$.

Using the above notation, Proposition 3.1 may be written,

Proposition 3.1 For $n \geq 4, E(n, 2)=\frac{n(n+1)}{6} \sqrt{\frac{n+1}{3(n-1)}}$. This upper bound is attained only when $\varepsilon$ is the maximal ellipse of a triangular slice of $S$ containing two of the vertices. 
Before giving the full proof of Proposition 3.1 we will examine a special case to show how the proof works.

When $k=2$ the conditions may be written,

$$
\begin{aligned}
& \text { (A) } \quad \theta x_{i}+\sqrt{x_{i}^{2}+y_{i}^{2}} \leq 1 \quad 1 \leq i \leq n+1 \\
& \text { (B) } \quad \sum_{i=1}^{n+1} x_{i}=\sum_{i=1}^{n+1} y_{i}=0
\end{aligned}
$$

for a sequence of vectors $\left\{\left(x_{i}, y_{i}\right)\right\}_{i=1}^{n+1}$ in $R^{2}, \theta \in R$, and we show

$$
D=\operatorname{Det}\left(\begin{array}{cc}
\sum_{i=1}^{n+1} x_{i}^{2} & \sum_{i=1}^{n+1} x_{i} y_{i} \\
\sum_{i=1}^{n+1} x_{i} y_{i} & \sum_{i=1}^{n+1} y_{i}^{2}
\end{array}\right) \leq \frac{(n+1)^{5}}{108(n-1)}
$$

The special case we consider is that of $\theta=1$.In this case the condition $(A)$ is,

$$
x_{i}+\sqrt{x_{i}^{2}+y_{i}^{2}} \leq 1 \quad 1 \leq i \leq n+1
$$

so

$$
y_{i}^{2} \leq 1-2 x_{i} \quad 1 \leq i \leq n+1
$$

Summing this inequality over $1 \leq i \leq n+1$ and using condition $(B)$ gives

$$
\sum_{i=1}^{n+1} y_{i}^{2} \leq n+1
$$

Hence

$$
\begin{aligned}
D & =\sum_{i=1}^{n+1} x_{i}^{2} \sum_{i=1}^{n+1} y_{i}^{2}-\left(\sum_{i=1}^{n+1} x_{i} y_{i}\right)^{2} \\
& \leq \sum_{i=1}^{n+1} x_{i}^{2} \sum_{i=1}^{n+1} y_{i}^{2} \\
& \leq(n+1) \sum_{i=1}^{n+1} x_{i}^{2} \quad(*)
\end{aligned}
$$


That is, we may estimate $D$ from above when $\sum_{i=1}^{n+1} x_{i}^{2}$ is 'small', and we now need to find an upper bound for $D$ when $\sum_{i=1}^{n+1} x_{i}^{2}$ is 'large'.

By condition $(A)$, for each $i, 1 \leq i \leq n+1$, we have $x_{i} \leq \frac{1}{2}$ so for any pair $1 \leq i<j \leq n+1$,

$$
0 \leq\left(y_{i}+y_{j}\right)^{2}\left(\frac{1}{2}-x_{i}\right)\left(\frac{1}{2}-x_{j}\right)
$$

After summing over all pairs $1 \leq i<j \leq n+1$ then applying condition $(B)$ this gives,

$$
-2 \sum_{1 \leq i<j \leq n+1} x_{i} x_{j} y_{i} y_{j} \leq \frac{n-1}{4} \sum_{i=1}^{n+1} y_{i}^{2}-\sum_{i=1}^{n+1} x_{i}^{2} y_{i}^{2}-\frac{n-3}{2} \sum_{i=1}^{n+1} x_{i} y_{i}^{2}
$$

We then apply this inequality to $D$,

$$
\begin{aligned}
D & =\sum_{i=1}^{n+1} x_{i}^{2} \sum_{i=1}^{n+1} y_{i}^{2}-\sum_{i=1}^{n+1} x_{i}^{2} y_{i}^{2}-2 \sum_{i<j} x_{i} x_{j} y_{i} y_{j} \\
& \leq \frac{n-1}{4} \sum_{i=1}^{n+1} y_{i}^{2}-\frac{n-3}{2} \sum_{i=1}^{n+1} x_{i} y_{i}^{2}+\sum_{i=1}^{n+1} x_{i}^{2} \sum_{i=1}^{n+1} y_{i}^{2}-2 \sum_{i=1}^{n+1} x_{i}^{2} y_{i}^{2}
\end{aligned}
$$

Also,

$$
\frac{1}{n} D \leq \frac{1}{n} \sum_{i=1}^{n+1} x_{i}^{2} \sum_{i=1}^{n+1} y_{i}^{2}
$$

so adding these final two inequalities we have

$$
\frac{n+1}{n} D \leq \sum_{i=1}^{n+1} y_{i}^{2}\left(\frac{n-1}{4}-\frac{n-3}{2} x_{i}-2 x_{i}^{2}+\frac{n+1}{n} \sum_{j=1}^{n+1} x_{j}^{2}\right)
$$

We next show that the bracketed term in (1) is positive so that we may use condition (A) to remove the $y_{i}$ s from the estimate completely .

For each $1 \leq j \leq n+1, x_{j} \leq \frac{1}{2}$, so by condition $(B)$,

$$
-\frac{1}{2} \leq \sum_{i \neq j} x_{i} \leq \frac{n}{2}
$$


Since the quadratic

$$
q(z)=\frac{n-1}{4}+\frac{n-3}{2} z-\frac{n^{2}-2 n-1}{n^{2}} z^{2}
$$

is positive on the interval $\left[-\frac{1}{2}, \frac{n}{2}\right]$ we have, for each $1 \leq j \leq n+1$,

$$
\frac{n-1}{4}+\frac{n-3}{2} \sum_{i \neq j} x_{i}-\frac{n^{2}-2 n-1}{n^{2}}\left(\sum_{i \neq j} x_{i}\right)^{2} \geq 0
$$

By the Cauchy-Schwarz inequality,

$$
\frac{n+1}{n} \sum_{i \neq j} x_{i}^{2} \geq \frac{n+1}{n^{2}}\left(\sum_{i \neq j} x_{i}\right)^{2}
$$

Adding (2) and (3),

$$
\frac{n-1}{4}+\frac{n-3}{2} \sum_{i \neq j} x_{i}-\frac{n-1}{n}\left(\sum_{i \neq j} x_{i}\right)^{2}+\frac{n+1}{n} \sum_{i \neq j} x_{i}^{2} \geq 0
$$

that is, for each $j$,

$$
\frac{n-1}{4}-\frac{n-3}{2} x_{j}+\frac{n+1}{n} \sum_{i=1}^{n+1} x_{i}^{2}-2 x_{j}^{2} \geq 0
$$

as required.

Applying condition $(A)$ to (1) gives

$$
\frac{n+1}{n} D \leq 4 \sum_{i=1}^{n+1} x_{i}^{3}+\left(n-5+\frac{(n+1)^{2}}{n}\right) \sum_{i=1}^{n+1} x_{i}^{2}+\frac{(n+1)(n-1)}{4}
$$

so we need to estimate $\sum_{i=1}^{n+1} x_{i}^{3}$ from above.

Again, since for each $i, x_{i} \leq \frac{1}{2}$, condition $(B)$ gives

$$
x_{i}+x_{j} \geq-\frac{n-1}{2}
$$


for every pair $1 \leq i<j \leq n+1$, and so

$$
\left(\frac{n-1}{2}+x_{i}+x_{j}\right)\left(\frac{1}{2}-x_{i}\right)\left(\frac{1}{2}-x_{j}\right) \geq 0
$$

After expanding this expression and summing over all pairs $1 \leq i<j \leq n+1$, we have

$$
\frac{n(n+1)(n-1)}{16}-\frac{3}{4}(n-1) \sum_{i=1}^{n+1} x_{i}^{2}-\sum_{i=1}^{n+1} x_{i}^{3} \geq 0
$$

which when applied to (4) gives

$$
\begin{aligned}
\frac{n+1}{n} D & \leq \frac{(n+1)^{2}(n-1)}{4}-\frac{(n+1)(n-1)}{n} \sum_{i=1}^{n+1} x_{i}^{2} \\
D & \leq \frac{n(n+1)(n-1)}{4}-(n-1) \sum_{i=1}^{n+1} x_{i}^{2}
\end{aligned}
$$

From earlier we have

$$
D \leq(n+1) \sum_{i=1}^{n+1} x_{i}^{2}
$$

so combining $(*)$ and $(* *)$,

$$
\begin{aligned}
D & \leq \frac{(n+1)^{2}(n-1)}{8} \\
& \leq \frac{(n+1)^{5}}{108(n-1)}
\end{aligned}
$$

with equality if and only if $n=5$. 
In this section we will prove Proposition 3.1 for $n \geq 5$, leaving $n=4$ for the final section of the chapter. The technical part of the proof of Proposition 3.1 is taken care of by the following theorem which generalises the special case of $\theta=1$ above,

Theorem 3.3 Let $n \geq 3, \theta \geq \frac{n-1}{n+1}$ and let $\left(x_{i}\right)_{i=1}^{n+1},\left(y_{i}\right)_{i=1}^{n+1}$ be sequences in $R$ satisfying

$$
\theta x_{i}+\sqrt{x_{i}^{2}+y_{i}^{2}} \leq 1 \quad 1 \leq i \leq n+1
$$

and

$$
\sum_{i=1}^{n+1} x_{i}=\sum_{i=1}^{n+1} y_{i}=0
$$

then,

$$
\operatorname{Det}\left(\begin{array}{cc}
\sum_{i=1}^{n+1} x_{i}^{2} & \sum_{i=1}^{n+1} x_{i} y_{i} \\
\sum_{i=1}^{n+1} x_{i} y_{i} & \sum_{i=1}^{n+1} y_{i}^{2}
\end{array}\right) \leq \frac{(n+1)^{2}(n-1)}{4(1+\theta)^{3}}((n+1) \theta-(n-3))
$$

\section{The Proof of Proposition 3.1}

We have two sequences $\left(x_{i}\right)_{i=1}^{n+1},\left(y_{i}\right)_{i=1}^{n+1}$ in $R$ and $\theta \in R$ satisfying

$$
\begin{aligned}
& \text { (A) } \quad \theta x_{i}+\sqrt{x_{i}^{2}+y_{i}^{2}} \leq 1 \quad 1 \leq i \leq n+1 \\
& \text { (B) } \quad \sum_{i=1}^{n+1} x_{i}=\sum_{i=1}^{n+1} y_{i}=0
\end{aligned}
$$

and show that

$$
D=\operatorname{Det}\left(\begin{array}{cc}
\sum_{i=1}^{n+1} x_{i}^{2} & \sum_{i=1}^{n+1} x_{i} y_{i} \\
\sum_{i=1}^{n+1} x_{i} y_{i} & \sum_{i=1}^{n+1} y_{i}^{2}
\end{array}\right) \leq \frac{(n+1)^{5}}{108(n-1)}
$$

As in the proof of the $k=1$ case we may take $\theta \geq 0$ and the proof breaks at $\theta=\frac{n-1}{n+1}$. 
CASE $1.0 \leq \theta \leq \frac{n-1}{n+1}$

By condition $(A)$, for each $i, 1 \leq i \leq n+1$

$$
y_{i}^{2} \leq\left(\theta^{2}-1\right) x_{i}^{2}-2 \theta x_{i}+1
$$

so summing over $i=1, \ldots, n+1$ and using condition $(B)$ gives

$$
\sum_{i=1}^{n+1} y_{i}^{2} \leq\left(\theta^{2}-1\right) \sum_{i=1}^{n+1} x_{i}^{2}+(n+1)
$$

Therefore,

$$
\begin{aligned}
D & =\sum_{i=1}^{n+1} x_{i}^{2} \sum_{i=1}^{n+1} y_{i}^{2}-\left(\sum_{i=1}^{n+1} x_{i} y_{i}\right)^{2} \\
& \leq \sum_{i=1}^{n+1} x_{i}^{2} \sum_{i=1}^{n+1} y_{i}^{2} \\
& \leq\left(\theta^{2}-1\right)\left(\sum_{i=1}^{n+1} x_{i}^{2}\right)^{2}+(n+1) \sum_{i=1}^{n+1} x_{i}^{2}
\end{aligned}
$$

Maximizing the right hand side of this final inequality with respect to $\sum_{i=1}^{n+1} x_{i}^{2}$, we have

$$
D \leq \frac{(n+1)^{2}}{4\left(1-\theta^{2}\right)}
$$

and since $0 \leq \theta \leq \frac{n-1}{n+1}, n \geq 5$,

$$
D<\frac{(n+1)^{5}}{108(n-1)}
$$

CAse 2. $\theta \geq \frac{n-1}{n+1}$

By Theorem 3.3

$$
D \leq \frac{(n+1)^{2}(n-1)}{4(1+\theta)^{3}}((n+1) \theta-(n-3))
$$


and this function attains its maximum at $\theta=\frac{2(n-2)}{n+1}$ so that

$$
D \leq \frac{(n+1)^{5}}{108(n-1)}
$$

as required.

Equality occurs in Proposition 3.1 if and only if $\theta=\frac{2(n-2)}{n+1}$ and the sequence $\left\{\left(x_{i}, y_{i}\right)\right\}_{1}^{n+1}$ consists of $n-1$ vectors of the form $\left(\frac{n+1}{3(n-1)}, 0\right)$ and 2 vectors $\left(-\frac{n+1}{6}, \frac{n+1}{2 \sqrt{3}}\right)$ and $\left(-\frac{n+1}{6},-\frac{n+1}{2 \sqrt{3}}\right)$. This occurs when all but two of the $u_{i}$ s project to the same point when projected onto the subspace spanned by $\varepsilon$. That is, the subspace spanned by $\varepsilon$ is perpendicular to an $(n-2)$-dimensional face of $S$.

3. The Proof of Theorem 3.3

Let $\left(x_{i}\right)_{i=1}^{n+1},\left(y_{i}\right)_{i=1}^{n+1}$ be sequences in $R$ and $\theta \geq \frac{n-1}{n+1}$ satisfying

(A) $\quad \theta x_{i}+\sqrt{x_{i}^{2}+y_{i}^{2}} \leq 1 \quad 1 \leq i \leq n+1$

(B) $\quad \sum_{i=1}^{n+1} x_{i}=\sum_{i=1}^{n+1} y_{i}=0$

We show that

$$
D=\operatorname{Det}\left(\begin{array}{cc}
\sum_{i=1}^{n+1} x_{i}^{2} & \sum_{i=1}^{n+1} x_{i} y_{i} \\
\sum_{i=1}^{n+1} x_{i} y_{i} & \sum_{i=1}^{n+1} y_{i}^{2}
\end{array}\right) \leq \frac{(n+1)^{2}(n-1)}{4(1+\theta)^{3}}((n+1) \theta-(n-3))
$$

By condition $(A)$, for every $i, 1 \leq i \leq n+1$,

$$
x_{i} \leq \frac{1}{1+\theta}
$$

so for any pair $i, j$, with $1 \leq i<j \leq n+1$,

$$
0 \leq\left(y_{i}+y_{j}\right)^{2}\left(\frac{1}{1+\theta}-x_{i}\right)\left(\frac{1}{1+\theta}-x_{j}\right)
$$

51 
After expanding this expression and summing it over all pairs, $1 \leq i<j \leq n+1$, then using condition $(B)$ where necessary we have

$$
-2 \sum_{1 \leq i<j \leq n+1} x_{i} x_{j} y_{i} y_{j} \leq \frac{n-1}{(1+\theta)^{2}} \sum_{i=1}^{n+1} y_{i}^{2}-\sum_{i=1}^{n+1} x_{i}^{2} y_{i}^{2}-\frac{n-3}{1+\theta} \sum_{i=1}^{n+1} x_{i} y_{i}^{2}
$$

We then apply this inequality to $D$,

$$
\begin{aligned}
D & =\sum_{i=1}^{n+1} x_{i}^{2} \sum_{i=1}^{n+1} y_{i}^{2}-\sum_{i=1}^{n+1} x_{i}^{2} y_{i}^{2}-2 \sum_{i<j} x_{i} x_{j} y_{i} y_{j} \\
& \leq \frac{n-1}{(1+\theta)^{2}} \sum_{i=1}^{n+1} y_{i}^{2}-\frac{n-3}{1+\theta} \sum_{i=1}^{n+1} x_{i} y_{i}^{2}+\sum_{i=1}^{n+1} x_{i}^{2} \sum_{i=1}^{n+1} y_{i}^{2}-2 \sum_{i=1}^{n+1} x_{i}^{2} y_{i}^{2}
\end{aligned}
$$

Also,

$$
\frac{1}{n} D \leq \frac{1}{n} \sum_{i=1}^{n+1} x_{i}^{2} \sum_{i=1}^{n+1} y_{i}^{2}
$$

so adding these final two inequalities we have

$$
\frac{n+1}{n} D \leq \sum_{i=1}^{n+1} y_{i}^{2}\left(\frac{n-1}{(1+\theta)^{2}}-\frac{n-3}{1+\theta} x_{i}-2 x_{i}^{2}+\frac{n+1}{n} \sum_{j=1}^{n+1} x_{j}^{2}\right)
$$

Since for every $1 \leq j \leq n+1$,

$$
x_{j} \leq \frac{1}{1+\theta}
$$

and $\sum_{i=1}^{n+1} x_{i}=0$ we have, for every $j$

$$
-\frac{1}{1+\theta} \leq \sum_{i \neq j} x_{i} \leq \frac{n}{1+\theta}
$$

The quadratic

$$
q(z)=\frac{n-1}{(1+\theta)^{2}}+\frac{n-3}{1+\theta} z-\frac{n^{2}-2 n-1}{n^{2}} z^{2}
$$


is positive on the interval $\left[-\frac{1}{1+\theta}, \frac{n}{1+\theta}\right]$ so we have, for each $1 \leq j \leq n+1$,

$$
\frac{n-1}{(1+\theta)^{2}}+\frac{n-3}{1+\theta} \sum_{i \neq j} x_{i}-\frac{n^{2}-2 n-1}{n^{2}}\left(\sum_{i \neq j} x_{i}\right)^{2} \geq 0
$$

By the Cauchy-Schwarz inequality,

$$
\frac{n+1}{n} \sum_{i \neq j} x_{i}^{2} \geq \frac{n+1}{n^{2}}\left(\sum_{i \neq j} x_{i}\right)^{2}
$$

Adding (3.2) and (3.3),

$$
\frac{n-1}{(1+\theta)^{2}}+\frac{n-3}{1+\theta} \sum_{i \neq j} x_{i}-\frac{n-1}{n}\left(\sum_{i \neq j} x_{i}\right)^{2}+\frac{n+1}{n} \sum_{i \neq j} x_{i}^{2} \geq 0
$$

that is, for each $j$,

$$
\frac{n-1}{(1+\theta)^{2}}-\frac{n-3}{1+\theta} x_{j}+\frac{n+1}{n} \sum_{i=1}^{n+1} x_{i}^{2}-2 x_{j}^{2} \geq 0
$$

This inequality means that we may apply condition $(A)$ in the form

$$
y_{i}^{2} \leq\left(\theta^{2}-1\right) x_{i}^{2}-2 \theta x_{i}+1
$$

for each $i$, to inequality (3.1) to obtain,

$$
\begin{aligned}
\frac{n+1}{n} D \leq & \left(\theta^{2}-1\right)\left(\frac{n+1}{n}\left(\sum_{i=1}^{n+1} x_{i}^{2}\right)^{2}-2 \sum_{i=1}^{n+1} x_{i}^{4}\right) \\
& +(4 \theta-(n-3)(\theta-1)) \sum_{i=1}^{n+1} x_{i}^{3} \\
& +\left(\frac{3(n-3) \theta-(n+1)}{1+\theta}+\frac{(n+1)^{2}}{n}\right) \sum_{i=1}^{n+1} x_{i}^{2} \\
& +\frac{(n+1)(n-1)}{(1+\theta)^{2}}
\end{aligned}
$$


The proof splits into three cases. The first, easy case is for $\sum_{i=1}^{n+1} x_{i}^{2} \leq \frac{(n+1)(n-1)}{2(1+\theta)^{2}}$ where a simple estimate for $D$ as the product of the main diagonal entries of the matrix is sufficient. The second and third cases are for $\sum_{i=1}^{n+1} x_{i}^{2} \geq \frac{(n+1)(n-1)}{2(1+\theta)^{2}}$ with either $\theta \geq 1$ or $\frac{n-1}{n+1} \leq \theta<1$ and these cases require the more complicated estimate for $D$ given by (3.4).

CASE 1. $\sum_{i=1}^{n+1} x_{i}^{2} \leq \frac{(n+1)(n-1)}{2(1+\theta)^{2}}$

By condition $(A)$, for each $i, 1 \leq i \leq n+1$

$$
y_{i}^{2} \leq\left(\theta^{2}-1\right) x_{i}^{2}-2 \theta x_{i}+1
$$

so summing over $i=1, \ldots, n+1$ and using condition $(B)$ gives

$$
\sum_{i=1}^{n+1} y_{i}^{2} \leq\left(\theta^{2}-1\right) \sum_{i=1}^{n+1} x_{i}^{2}+(n+1)
$$

Therefore,

$$
\begin{aligned}
D & =\sum_{i=1}^{n+1} x_{i}^{2} \sum_{i=1}^{n+1} y_{i}^{2}-\left(\sum_{i=1}^{n+1} x_{i} y_{i}\right)^{2} \\
& \leq \sum_{i=1}^{n+1} x_{i}^{2} \sum_{i=1}^{n+1} y_{i}^{2} \\
& \leq\left(\theta^{2}-1\right)\left(\sum_{i=1}^{n+1} x_{i}^{2}\right)^{2}+(n+1) \sum_{i=1}^{n+1} x_{i}^{2}
\end{aligned}
$$

Since $\theta \geq \frac{n-1}{n+1}$ this last expression is an increasing function of $\sum_{i=1}^{n+1} x_{i}^{2}$ on the interval $\left[0, \frac{(n-1)(n+1)}{2(1+\theta)^{2}}\right]$ and therefore

$$
\begin{aligned}
D & \leq\left(\theta^{2}-1\right)\left(\frac{(n-1)(n+1)}{2(1+\theta)^{2}}\right)^{2}+\frac{(n-1)(n+1)^{2}}{2(1+\theta)^{2}} \\
& =\frac{(n+1)^{2}(n-1)}{4(1+\theta)^{3}}((n+1) \theta-(n-3))
\end{aligned}
$$


CASE 2. $\sum_{i=1}^{n+1} x_{i}^{2} \geq \frac{(n+1)(n-1)}{2(1+\theta)^{2}}, \frac{n-1}{n+1} \leq \theta<1$

By condition $(A)$ for every $i, 1 \leq i \leq n+1$, we have $x_{i} \leq \frac{1}{1+\theta}$ so, by condition $(B)$, for every pair $1 \leq i<j \leq n+1$,

$$
-\frac{(n-1)}{1+\theta} \leq x_{i}+x_{j}
$$

Multiplying both sides of this inequality by $\left(1-\theta^{2}\right)$ and adding $2 \theta$ gives

$$
(n+1) \theta-(n-1) \leq\left(1-\theta^{2}\right)\left(x_{i}+x_{j}\right)+2 \theta .
$$

As $\theta \geq \frac{n-1}{n+1}$ the left hand side of this inequality is non-negative, so for any pair $1 \leq i<j \leq n+1$

$$
0 \leq\left(\left(1-\theta^{2}\right)\left(x_{i}+x_{j}\right)+2 \theta\right)^{2}-((n+1) \theta-(n-1))^{2}
$$

and therefore

$0 \leq \frac{1}{1-\theta^{2}}\left[\left(\left(1-\theta^{2}\right)\left(x_{i}+x_{j}\right)+2 \theta\right)^{2}-((n+1) \theta-(n-1))^{2}\right]\left[\frac{1}{1+\theta}-x_{i}\right]\left[\frac{1}{1+\theta}-x_{j}\right]$

Expanding this expression and summing over all pairs $1 \leq i<j \leq n+1$, using condition $(B)$ where necessary yields the following inequality

$$
\begin{aligned}
0 \leq & \left(1-\theta^{2}\right)\left(\left(\sum_{i=1}^{n+1} x_{i}^{2}\right)^{2}-2 \sum_{i=1}^{n+1} x_{i}^{4}\right)+((n-3)(\theta-1)-4 \theta) \sum_{i=1}^{n+1} x_{i}^{3} \\
& +\frac{n-1}{2(1+\theta)}((n+1)-(n+13) \theta) \sum_{i=1}^{n+1} x_{i}^{2} \\
& +\frac{n(n+1)(n-1)((n+3) \theta-(n-1))}{2(1+\theta)^{3}}
\end{aligned}
$$


Adding (3.4) and (3.5),

$$
\begin{aligned}
\frac{n+1}{n} D \leq & \frac{1}{n}\left(\theta^{2}-1\right)\left(\sum_{i=1}^{n+1} x_{i}^{2}\right)^{2} \\
& +\frac{(n+1)\left(n^{2}-n+2\right)-(n+1)\left(n^{2}+3 n-2\right) \theta}{2 n(1+\theta)} \sum_{i=1}^{n+1} x_{i}^{2} \\
& +\frac{(n+1)^{2}(n-1)((n+2) \theta-(n-2))}{2(1+\theta)^{3}}
\end{aligned}
$$

Since $n \geq 3$ and $\frac{n-1}{n+1} \leq \theta<1$ the coefficients of $\sum_{i=1}^{n+1} x_{i}^{2}$ and $\left(\sum_{i=1}^{n+1} x_{i}^{2}\right)^{2}$ are both negative, so using

$$
\sum_{i=1}^{n+1} x_{i}^{2} \geq \frac{(n+1)(n-1)}{2(1+\theta)^{2}}
$$

we have in the last inequality,

$$
D \leq \frac{(n+1)^{2}(n-1)}{4(1+\theta)^{3}}((n+1) \theta-(n-3))
$$

CASE 3. $\sum_{i=1}^{n+1} x_{i}^{2} \geq \frac{(n+1)(n-1)}{2(1+\theta)^{2}}, \theta \geq 1$

As in case 2 , for each pair $1 \leq i<j \leq n+1$

$$
-\frac{(n-1)}{1+\theta} \leq x_{i}+x_{j} \leq \frac{2}{1+\theta}
$$

that is

$$
-\frac{(n+1)}{2(1+\theta)} \leq x_{i}+x_{j}+\frac{(n-3)}{2(1+\theta)} \leq \frac{(n+1)}{2(1+\theta)}
$$

Squaring this inequality,

$$
0 \leq\left(x_{i}+x_{j}+\frac{(n-3)}{2(1+\theta)}\right)^{2} \leq \frac{(n+1)^{2}}{4(1+\theta)^{2}}
$$


From this and the fact that for each $i, x_{i} \leq \frac{1}{1+\theta}$ it follows that for every pair $1 \leq i<j \leq n+1$

$$
0 \leq\left[\frac{1}{1+\theta}-x_{i}\right]\left[\frac{1}{1+\theta}-x_{j}\right]\left[\frac{(n+1)^{2}}{4(1+\theta)^{2}}-\left(x_{i}+x_{j}+\frac{(n-3)}{2(1+\theta)}\right)^{2}\right]
$$

and

$$
0 \leq\left[x_{i}+x_{j}+\frac{(n-3)}{2(1+\theta)}\right]^{2}\left[\frac{(n+1)^{2}}{4(1+\theta)^{2}}-\left(x_{i}+x_{j}+\frac{(n-3)}{2(1+\theta)}\right)^{2}\right]
$$

Expanding and summing over all pairs $1 \leq i<j \leq n+1$ each of these expressions, using condition $(B)$ where necessary gives

$$
\begin{aligned}
\left(\sum_{i=1}^{n+1} x_{i}^{2}\right)^{2}-2 \sum_{i=1}^{n+1} x_{i}^{4} \leq & \frac{n(n+1)(n-1)}{(1+\theta)^{4}}+\frac{(n-5)(n-1)}{(1+\theta)^{2}} \sum_{i=1}^{n+1} x_{i}^{2} \\
& +\frac{2(n-3)}{1+\theta} \sum_{i=1}^{n+1} x_{i}^{3}
\end{aligned}
$$

and

$$
\begin{aligned}
3\left(\sum_{i=1}^{n+1} x_{i}^{2}\right)^{2}+(n-7) \sum_{i=1}^{n+1} x_{i}^{4} \leq & \frac{n(n+1)(n-1)(n-3)^{2}}{4(1+\theta)^{4}} \\
& -\frac{(n-1)\left(5 n^{2}-38 n+53\right)}{4(1+\theta)^{2}} \sum_{i=1}^{n+1} x_{i}^{2} \\
& -\frac{2(n-3)^{2}}{1+\theta} \sum_{i=1}^{n+1} x_{i}^{3}
\end{aligned}
$$

Adding together $\frac{n^{2}-7}{n(n-1)}$ times (3.6) and $\frac{2}{n(n-1)}$ times (3.7), then multiplying the result by $\left(\theta^{2}-1\right)$,

$$
\begin{aligned}
\left(\theta^{2}-1\right)\left(\frac{n+1}{n}\left(\sum_{i=1}^{n+1} x_{i}^{2}\right)^{2}-2 \sum_{i=1}^{n+1} x_{i}^{4}\right) \leq & \frac{2(n-3)\left(n^{2}-2 n-1\right)(\theta-1)}{n(n-1)} \sum_{i=1}^{n+1} x_{i}^{3} \\
& +\frac{\left(2 n^{3}-15 n^{2}+24 n+17\right)(\theta-1)}{2 n(1+\theta)} \sum_{i=1}^{n+1} x_{i}^{2} \\
& +\frac{(n+1)\left(3 n^{2}-6 n-5\right)(\theta-1)}{2(1+\theta)^{3}}
\end{aligned}
$$


Adding (3.4) and (3.8) and multiplying by $\frac{n}{n+1}$,

$$
\begin{aligned}
D \leq & \frac{(n+1)\left(n^{2}-3 n+6\right) \theta-(n-3)\left(n^{2}-3 n-2\right)}{(n+1)(n-1)} \sum_{i=1}^{n+1} x_{i}^{3} \\
& +\frac{(n+1)\left(2 n^{2}-9 n+19\right) \theta-(n-3)(2 n+1)(n-5)}{2(n+1)(1+\theta)} \sum_{i=1}^{n+1} x_{i}^{2} \\
& +\frac{n(n+1)(3 n-7) \theta-n(3 n+1)(n-3)}{2(1+\theta)^{3}}
\end{aligned}
$$

The coefficient of $\sum_{i=1}^{n+1} x_{i}^{3}$ is positive so we estimate $\sum_{i=1}^{n+1} x_{i}^{3}$ from above as follows.

For every pair $1 \leq i<j \leq n+1$ we have

$$
0 \leq\left(\frac{n-1}{1+\theta}+x_{i}+x_{j}\right)\left(\frac{1}{1+\theta}-x_{i}\right)\left(\frac{1}{1+\theta}-x_{j}\right)
$$

Expanding and summing this over all pairs $1 \leq i<j \leq n+1$ gives

$$
\sum_{i=1}^{n+1} x_{i}^{3} \leq \frac{n(n+1)(n-1)}{2(1+\theta)^{3}}-\frac{3(n-1)}{2(1+\theta)} \sum_{i=1}^{n+1} x_{i}^{2}
$$

From (3.9) and (3.10) we have

$$
D \leq \frac{n(n+1)(n-1)}{2(1+\theta)^{3}}((n+1) \theta-(n-3))-\frac{(n-1)}{2(1+\theta)}((n+1) \theta-(n-3)) \sum_{i=1}^{n+1} x_{i}^{2}
$$

and since $\sum_{i=1}^{n+1} x_{i}^{2} \geq \frac{(n+1)(n-1)}{2(1+\theta)^{2}}$

$$
D \leq \frac{(n+1)^{2}(n-1)}{4(1+\theta)^{3}}((n+1) \theta-(n-3))
$$

Equality occurs in Theorem 3.3 if and only if the sequence $\left\{\left(x_{i}, y_{i}\right)\right\}_{1}^{n+1}$ consists of $n-1$ vectors of the form $\left(\frac{1}{1+\theta}, 0\right)$ and 2 vectors $\left(-\frac{n-1}{2(1+\theta)}, \frac{1}{2} \sqrt{\frac{(n+1)((n+1) \theta-(n-3))}{1+\theta}}\right)$ and $\left(-\frac{n-1}{2(1+\theta)},-\frac{1}{2} \sqrt{\frac{(n+1)((n+1) \theta-(n-3))}{1+\theta}}\right)$. 


\section{The Proof of Proposition 3.1 when $n=4$}

Let $\left(x_{i}\right)_{i=1}^{5},\left(y_{i}\right)_{i=1}^{5}$ be sequences in $R$ and $\theta \in R$ satisfying

$$
\begin{array}{lll}
\text { (A) } & \theta x_{i}+\sqrt{x_{i}^{2}+y_{i}^{2}} \leq 1 & 1 \leq i \leq 5 \\
\text { (B) } & \sum_{i=1}^{5} x_{i}=\sum_{i=1}^{5} y_{i}=0 &
\end{array}
$$

We show that

$$
D=\operatorname{Det}\left(\begin{array}{cc}
\sum_{i=1}^{5} x_{i}^{2} & \sum_{i=1}^{5} x_{i} y_{i} \\
\sum_{i=1}^{5} x_{i} y_{i} & \sum_{i=1}^{5} y_{i}^{2}
\end{array}\right) \leq \frac{(n+1)^{5}}{108(n-1)}=\frac{3125}{324}
$$

When $n \geq 5$ the proof of Proposition 3.1 was split into two cases depending on the value of $\theta$. For $n=4$ we break the proof into three cases $0 \leq \theta \leq \frac{1}{2}, \theta \geq \frac{3}{5}$ and $\frac{1}{2}<\theta<\frac{3}{5}$, the last case being the part that needs a new proof.

CASE $1.0 \leq \theta \leq \frac{1}{2}$

The proof is identical to the $n \geq 5$ case. Using both conditions $(A)$ and $(B)$,

$$
\begin{aligned}
\sum_{i=1}^{5} y_{i}^{2} & \leq\left(\theta^{2}-1\right) \sum_{i=1}^{5} x_{i}^{2}-2 \theta \sum_{i=1}^{5} x_{i}+5 \\
& =\left(\theta^{2}-1\right) \sum_{i=1}^{5} x_{i}^{2}+5
\end{aligned}
$$

so that

$$
\begin{aligned}
D & =\sum_{i=1}^{5} x_{i}^{2} \sum_{i=1}^{5} y_{i}^{2}-\left(\sum_{i=1}^{5} x_{i} y_{i}\right)^{2} \\
& \leq \sum_{i=1}^{5} x_{i}^{2} \sum_{i=1}^{5} y_{i}^{2} \\
& \leq\left(\theta^{2}-1\right)\left(\sum_{i=1}^{5} x_{i}^{2}\right)^{2}+5 \sum_{i=1}^{5} x_{i}^{2} \\
& \leq \frac{25}{4\left(1-\theta^{2}\right)}
\end{aligned}
$$




$$
\begin{aligned}
& \leq \frac{25}{3} \\
& <\frac{3125}{324}
\end{aligned}
$$

CAse 2. $\theta \geq \frac{3}{5}$

The proof is identical to the $n \geq 5$ case. By Theorem 3.3 , when $n=4$ we have

$$
D \leq \frac{75(5 \theta-1)}{4(1+\theta)^{3}} \leq \frac{3125}{324}
$$

with equality if and only if when $\theta=\frac{4}{5}$ and $\left\{\left(x_{i}, y_{i}\right)\right\}_{1}^{5}$ consists of 3 vectors of the form $\left(\frac{5}{9}, 0\right)$ and 2 vectors $\left(-\frac{5}{6}, \frac{5}{2 \sqrt{3}}\right)$ and $\left(-\frac{5}{6},-\frac{5}{2 \sqrt{3}}\right)$.

CASE 3. $\frac{1}{2}<\theta<\frac{3}{5}$

By condition $(A)$, for every $i, 1 \leq i \leq 5$,

$$
x_{i} \leq \frac{1}{1+\theta}
$$

so for any pair $i, j$, with $1 \leq i<j \leq 5$,

$$
0 \leq\left(y_{i}+y_{j}\right)^{2}\left(\frac{1}{1+\theta}-x_{i}\right)\left(\frac{1}{1+\theta}-x_{j}\right)
$$

After expanding this expression and summing it over all pairs, $1 \leq i<j \leq 5$, then using condition $(B)$ where necessary we have

$$
-2 \sum_{1 \leq i<j \leq 5} x_{i} x_{j} y_{i} y_{j} \leq \frac{3}{(1+\theta)^{2}} \sum_{i=1}^{5} y_{i}^{2}-\sum_{i=1}^{5} x_{i}^{2} y_{i}^{2}-\frac{1}{1+\theta} \sum_{i=1}^{5} x_{i} y_{i}^{2}
$$

We then apply this inequality to $D$, 


$$
\begin{aligned}
D= & \sum_{i=1}^{5} x_{i}^{2} \sum_{i=1}^{5} y_{i}^{2}-\sum_{i=1}^{5} x_{i}^{2} y_{i}^{2}-2 \sum_{i<j} x_{i} x_{j} y_{i} y_{j} \\
\leq & \frac{3}{(1+\theta)^{2}} \sum_{i=1}^{5} y_{i}^{2}-\frac{1}{1+\theta} \sum_{i=1}^{5} x_{i} y_{i}^{2}+\sum_{i=1}^{5} x_{i}^{2} \sum_{i=1}^{5} y_{i}^{2}-2 \sum_{i=1}^{5} x_{i}^{2} y_{i}^{2} \\
= & \frac{3}{(1+\theta)^{2}} \sum_{i=1}^{5} y_{i}^{2}-\frac{1}{1+\theta} \sum_{i=1}^{5} x_{i} y_{i}^{2}-\sum_{i=1}^{5} x_{i}^{2} \sum_{i=1}^{5} y_{i}^{2} \\
& +2\left(\sum_{i=1}^{5} y_{i}^{2}\left(\sum_{j=1}^{5} x_{j}^{2}-x_{i}^{2}\right)\right)
\end{aligned}
$$

Using condition $(A)$ in the form, for each $1 \leq i \leq 5$,

$$
y_{i}^{2} \leq\left(\theta^{2}-1\right) x_{i}^{2}-2 \theta x_{i}+1
$$

gives

$$
\begin{aligned}
D \leq & \frac{3}{(1+\theta)^{2}} \sum_{i=1}^{5} y_{i}^{2}-\frac{1}{1+\theta} \sum_{i=1}^{5} x_{i} y_{i}^{2}-\sum_{i=1}^{5} x_{i}^{2} \sum_{i=1}^{5} y_{i}^{2} \\
& +2\left(\left(\theta^{2}-1\right)\left(\sum_{i=1}^{5} x_{i}^{2}\right)^{2}-\left(\theta^{2}-1\right) \sum_{i=1}^{5} x_{i}^{4}+2 \theta \sum_{i=1}^{5} x_{i}^{3}+4 \sum_{i=1}^{5} x_{i}^{2}\right)
\end{aligned}
$$

Since for each $i, x_{i} \leq \frac{1}{1+\theta}$, condition $(B)$ gives

$$
-\frac{3}{1+\theta} \leq x_{i}+x_{j} \leq \frac{2}{1+\theta}
$$

for each pair $1 \leq i<j \leq 5$, that is

$$
5 \theta-3 \leq\left(1-\theta^{2}\right)\left(x_{i}+x_{j}\right)+2 \theta
$$

Squaring the right hand side of this inequality and dividing by $1-\theta^{2}$ gives us

$$
0 \leq\left(1-\theta^{2}\right)\left(x_{i}+x_{j}\right)^{2}+4 \theta\left(x_{i}+x_{j}\right)+\frac{4 \theta^{2}}{1-\theta^{2}}
$$


for every pair $1 \leq i<j \leq 5$.

Therefore

$$
0 \leq 2\left[\left(1-\theta^{2}\right)\left(x_{i}+x_{j}\right)^{2}+4 \theta\left(x_{i}+x_{j}\right)+\frac{4 \theta^{2}}{1-\theta^{2}}\right]\left[\frac{1}{1+\theta}-x_{i}\right]\left[\frac{1}{1+\theta}-x_{j}\right]
$$

Expanding this inequality and summing over all pairs $1 \leq i<j \leq 5$ yields

$$
\begin{aligned}
2\left(\theta^{2}-1\right)\left(\left(\sum_{i=1}^{5} x_{i}^{2}\right)^{2}-\sum_{i=1}^{5} x_{i}^{4}\right)+4 \theta \sum_{i=1}^{5} x_{i}^{3} \leq & \left(\theta^{2}-1\right)\left(\sum_{i=1}^{5} x_{i}^{2}\right)^{2} \\
& +(\theta-1) \sum_{i=1}^{5} x_{i}^{3} \\
& +\frac{13 \theta^{2}-18 \theta+3}{1-\theta^{2}} \sum_{i=1}^{5} x_{i}^{2} \\
& +\frac{40 \theta^{2}}{(1-\theta)(1+\theta)^{3}}
\end{aligned}
$$

Also, using the fact that for each $1 \leq i \leq 5$,

$$
x_{i} \leq \frac{1}{1+\theta}
$$

and

$$
y_{i}^{2} \leq\left(\theta^{2}-1\right) x_{i}^{2}-2 \theta x_{i}+1
$$

we have

$$
\frac{1}{1+\theta} \sum_{i=1}^{5} y_{i}^{2}\left(\frac{1}{1+\theta}-x_{i}\right) \leq \frac{1}{1+\theta} \sum_{i=1}^{5}\left(\left(\theta^{2}-1\right) x_{i}^{2}-2 \theta x_{i}+1\right)\left(\frac{1}{1+\theta}-x_{i}\right)
$$

Rearranging this inequality gives 


$$
\begin{aligned}
(\theta-1) \sum_{i=1}^{5} x_{i}^{3}-\frac{1}{1+\theta} \sum_{i=1}^{5} x_{i} y_{i}^{2} \leq & \frac{5}{(1+\theta)^{2}}-\frac{1}{(1+\theta)^{2}} \sum_{i=1}^{5} y_{i}^{2} \\
& +\frac{3 \theta-1}{1+\theta} \sum_{i=1}^{5} x_{i}^{2}
\end{aligned}
$$

Adding inequalities (3.11), (3.12), (3.13) and using

$$
\frac{2}{(1+\theta)^{2}} \sum_{i=1}^{5} y_{i}^{2} \leq \frac{2(\theta-1)}{(1+\theta)} \sum_{i=1}^{5} x_{i}^{2}+\frac{10}{(1+\theta)^{2}}
$$

we have

$$
D \leq\left(\theta^{2}-1\right)\left(\sum_{i=1}^{5} x_{i}^{2}\right)^{2}-\sum_{i=1}^{5} x_{i}^{2} \sum_{i=1}^{5} y_{i}^{2}+\frac{8-10 \theta}{1-\theta^{2}} \sum_{i=1}^{5} x_{i}^{2}+\frac{15+25 \theta^{2}}{(1-\theta)(1+\theta)^{3}}
$$

Combining this with

$$
D \leq \sum_{i=1}^{5} x_{i}^{2} \sum_{i=1}^{5} y_{i}^{2}
$$

we have

$$
D \leq \frac{1}{2}\left(\theta^{2}-1\right)\left(\sum_{i=1}^{5} x_{i}^{2}\right)^{2}+\frac{4-5 \theta}{1-\theta^{2}} \sum_{i=1}^{5} x_{i}^{2}+\frac{15+25 \theta^{2}}{2(1-\theta)(1+\theta)^{3}}
$$

This final function has its maximum when $\sum_{i=1}^{5} x_{i}^{2}=\frac{4-5 \theta}{\left(1-\theta^{2}\right)^{2}}$.

Now when $\frac{1}{2}<\theta<\frac{3}{5}$,

$$
\frac{4-5 \theta}{\left(1-\theta^{2}\right)^{2}} \leq \frac{15}{2(1+\theta)^{2}}
$$

so that if $\sum_{i=1}^{5} x_{i}^{2} \geq \frac{15}{2(1+\theta)^{2}}$ then

$$
\begin{aligned}
D & \leq \frac{-125 \theta^{2}+150 \theta+75}{8(1-\theta)(1+\theta)^{3}} \\
& <\frac{3125}{324}
\end{aligned}
$$


Finally, if $\sum_{i=1}^{5} x_{i}^{2} \leq \frac{15}{2(1+\theta)^{2}}$ we have

$$
\begin{aligned}
D & \leq \sum_{i=1}^{5} x_{i}^{2} \sum_{i=1}^{5} y_{i}^{2} \\
& \leq\left(\theta^{2}-1\right)\left(\sum_{i=1}^{5} x_{i}^{2}\right)^{2}+5 \sum_{i=1}^{5} x_{i}^{2}
\end{aligned}
$$

which has its maximum at

$$
\sum_{i=1}^{5} x_{i}^{2}=\frac{5}{2\left(1-\theta^{2}\right)} \geq \frac{15}{2(1+\theta)^{2}}
$$

because $\theta \geq \frac{1}{2}$. Therefore, since $\frac{1}{2}<\theta<\frac{3}{5}$, (3.14) gives

$$
D \leq \frac{375 \theta-75}{(1+\theta)^{3}}<\frac{3125}{324}
$$




\section{Chapter 4}

In [8] Filliman claims that if $P^{*}$ is a $k$-dimensional critical central section (with respect to volume) of a regular $n$-simplex $S$ then the following conditions hold,

(a) The centroid of $P^{*}$ coincides with the centroid of $S$.

(b) Each facet of $P^{*}$ is normal to the line from its centroid to the centroid of $S$.

and that these conditions are sufficient to prove that the minimal 2-dimensional sections of $\mathrm{S}$ are equilateral triangles.

However, it appears that the conditions (a) and (b) are not quite correct. There are critical sections where neither condition holds.

Figure 4.1 shows a plot of the volume of 1-codimensional central sections of a regular 3-simplex, $S$, as a function of the point where the normal to the slice passing through the centroid of $S$ intersects one of the facets of $S$. The maximum values of the function, corresponding to sections that contain 2 vertices of $S$, can clearly be seen, as can the minimal values, corresponding to sections parallel to a facet of $S$. Also there are local minima corresponding to square sections. 


$$
\Delta
$$


The function is not differentiable at the maximum points, but it is differentiable at the local and global minima, where both of Filliman's results hold. However the function has saddle points where (a) and (b) do not hold.

If we take $S$ to be the 3 -simplex with edge length $\sqrt{2}$ defined in Chapter 1 , then the saddle points correspond to sections normal to vectors such as,

$$
\left(-\frac{1}{4},-\frac{1}{4}, \frac{1}{4}+\alpha, \frac{1}{4}-\alpha\right)
$$

where

$$
\alpha=\frac{\sqrt{\sqrt{33}-5}}{4}
$$

We may show this as follows.

As in Chapter 1 let a be a unit vector and $H$ be the 3-dimensional subspace of $R^{4}$ perpendicular to a.

To find the critical points of $\operatorname{Vol}_{2}(H \cap S)$ we recall the formula of Chapter 1 and find the critical points of

$$
2 \pi \operatorname{Vol}_{2}(H \bigcap S)=\int_{-\infty}^{\infty} \prod_{j=1}^{4} \frac{1}{1+i a_{j} t} d t
$$

under the conditions,

$$
\sum_{j=1}^{4} a_{j}=0 \quad \quad \sum_{j=1}^{4} a_{j}^{2}=1
$$

To ensure that the volume is differentiable we assume that all the $a_{j}$ are non-zero. Set

$$
f\left(a_{1}, a_{2}, a_{3}, a_{4}\right)=\int_{-\infty}^{\infty} \prod_{j=1}^{4} \frac{1}{1+i a_{j} t} d t+\lambda \sum_{j=1}^{4} a_{j}+\mu \sum_{j=1}^{4} a_{j}^{2}
$$


At the critical points,

$$
\frac{\partial f}{\partial a_{k}}=0 \quad k=1, \ldots, 4
$$

that is

$$
\int_{-\infty}^{\infty} \frac{-i t}{1+i a_{k} t} \prod_{j=1}^{4} \frac{1}{1+i a_{j} t} d t+\lambda+2 \mu a_{k}=0
$$

for each $1 \leq k \leq 4$

If all the $a_{j}$ are non-zero then the conditions may be multiplied by $a_{k}$ to give

$$
\int_{-\infty}^{\infty} \frac{-i a_{k} t}{1+i a_{k} t} \prod_{j=1}^{4} \frac{1}{1+i a_{j} t} d t+\lambda a_{k}+2 \mu a_{k}^{2}=0
$$

or , writing $M=2 \pi \operatorname{Vol}_{2}(H \cap S)$,

$$
-M+\int_{-\infty}^{\infty} \frac{1}{1+i a_{k} t} \prod_{j=1}^{4} \frac{1}{1+i a_{j} t} d t+\lambda a_{k}+2 \mu a_{k}^{2}=0
$$

for each $1 \leq k \leq 4$.

Now if,

$$
f(x)= \begin{cases}e^{-x} & \text { if } x \geq 0 \\ 0 & \text { otherwise }\end{cases}
$$

then the Fourier Transform of

$$
r_{k}(s)=\int_{H+s \mathbf{a}}<\mathbf{x}, \mathbf{e}_{k}>\prod_{j=1}^{4} f\left(<\mathbf{x}, \mathbf{e}_{j}>\right) d V o l_{H}
$$

is

$$
\hat{r}_{k}(t)=\frac{1}{1+i a_{k} t} \prod_{j=1}^{4} \frac{1}{1+i a_{j} t}
$$

so that

$$
\int_{-\infty}^{\infty} \hat{r}_{k}(t) d t=2 \pi r_{k}(0)
$$




$$
=2 \pi \int_{H}<\mathbf{x}, \mathbf{e}_{k}>\prod_{j=1}^{4} f\left(<\mathbf{x}, \mathbf{e}_{j}>\right) d V o l_{H}
$$

and as we saw in Chapter 2, this may be written as

$$
\int_{-\infty}^{\infty} \hat{r}_{k}(t) d t=3 c_{k} 2 \pi \int_{H} \prod_{j=1}^{4} f\left(<\mathbf{x}, \mathbf{e}_{j}>\right) d V o l_{H}
$$

where $c_{k}$ is the $k$-th coordinate of the centroid of $H \cap S$.

Therefore,

$$
\int_{-\infty}^{\infty} \hat{r}_{k}(t) d t=3 c_{k} M
$$

and the critical conditions are,

$$
-M+3 c_{k} M+\lambda a_{k}+2 \mu a_{k}^{2}=0
$$

for each $1 \leq k \leq 4$

As $\left(c_{1}, c_{2}, c_{3}, c_{4}\right)$ lies in $H \cap S$,

$$
\sum_{j=1}^{4} c_{j}=1
$$

and

$$
\sum_{j=1}^{4} a_{j} c_{j}=0
$$

so summing (4.1) over $k=1, \ldots, 4$ gives

$$
2 \mu=M
$$

and multiplying (4.1) by $a_{k}$ then summing gives

$$
\lambda=-M \sum_{k=1}^{4} a_{k}^{3}
$$


The critical conditions are therefore,

$$
c_{j}=\frac{1}{3}\left(1-a_{j}^{2}+a_{j}\left(\sum_{k=1}^{4} a_{k}^{3}\right)\right)
$$

for each $1 \leq j \leq 4$

If $\mathrm{a}$ is the unit vector in the direction $\left(-\frac{1}{4},-\frac{1}{4}, x, \frac{1}{2}-x\right)$, where $0<x<\frac{1}{2}$, then from (4.2) the critical conditions are

$$
\begin{aligned}
c_{1}=c_{2} & =\frac{4 x^{4}-4 x^{3}+2 x^{2}-\frac{1}{2} x+\frac{3}{32}}{3\left(\frac{3}{8}-x+2 x^{2}\right)^{2}} \\
c_{3} & =\frac{2 x^{4}-\frac{3}{2} x^{3}+\frac{11}{8} x^{2}-\frac{21}{32} x+\frac{9}{64}}{3\left(\frac{3}{8}-x+2 x^{2}\right)^{2}} \\
c_{4} & =\frac{2 x^{4}-\frac{5}{2} x^{3}+\frac{17}{8} x^{2}-\frac{19}{32} x+\frac{3}{32}}{3\left(\frac{3}{8}-x+2 x^{2}\right)^{2}}
\end{aligned}
$$

and the slice has vertices

$$
\begin{aligned}
& \left(\frac{4 x}{1+4 x}, 0, \frac{1}{1+4 x}, 0\right) \\
& \left(0, \frac{4 x}{1+4 x}, \frac{1}{1+4 x}, 0\right) \\
& \left(\frac{2-4 x}{3-4 x}, 0,0, \frac{1}{3-4 x}\right) \\
& \left(0, \frac{2-4 x}{3-4 x}, 0, \frac{1}{3-4 x}\right)
\end{aligned}
$$

It is easily checked that the critical conditions above, giving $\left(c_{1}, c_{2}, c_{3}, c_{4}\right)$, the centroid of the slice, are satisfied if and only if either $x=\frac{1}{4}$ or $x$ satisfies

$$
\frac{3}{16}-6 x+16 x^{2}-16 x^{3}+16 x^{4}=0
$$

that is,

$$
x=\frac{1}{4} \pm \frac{\sqrt{\sqrt{33}-5}}{4}
$$


but $c_{1}=c_{2}=c_{3}=c_{4}=\frac{1}{4}$ if and only if $x=\frac{1}{4}$. So we have a critical point that does not satisfy Filliman's conditions.

It is not hard to see that if Filliman's result (a) were correct then we could solve the problem of the minimal 1-codimensional central section of the regular $n$-simplex, since the $n$-dimensional version of the critical conditions derived above is

$$
c_{j}=\frac{1}{n}\left(1-a_{j}^{2}+a_{j}\left(\sum_{k=1}^{n+1} a_{k}^{3}\right)\right)
$$

for each $1 \leq j \leq n+1$, (by induction we can assume that all the $a_{j}$ are non-zero).

Applying (a) we see that all the $c_{j}$ are equal so that all the $a_{j}$ must satisfy the same quadratic equation. Therefore, for some $1 \leq k \leq n, k$ of the $a_{j}$ are equal to

$$
\sqrt{\frac{n+1-k}{k(n+1)}}
$$

and the remainder are equal to

$$
-\sqrt{\frac{k}{(n+1)(n-k+1)}}
$$

so that

$$
\begin{aligned}
\frac{(n-1) !}{\sqrt{n+1}} \operatorname{Vol}_{n-1}(H \bigcap S) & =\frac{1}{2 \pi} \int_{-\infty}^{\infty} \prod_{j=1}^{n+1} \frac{1}{1+i a_{j} t} d t \\
& =\frac{(n-1) !}{(n-k) !(k-1) !} \frac{\sqrt{n+1}(n+1)^{n}}{\sqrt{k}} \frac{k^{k}}{\sqrt{n-k+1}}
\end{aligned}
$$

We now show that this last function is minimised when $k=1$ or $k=n$. Let

$$
h(k)=\frac{(n-1) !}{(n-k) !(k-1) !} \frac{\sqrt{n+1}}{(n+1)^{n}} \frac{k^{k}}{\sqrt{k}} \frac{(n-k+1)^{n-k+1}}{\sqrt{n-k+1}}
$$


$h$ is symmetric on the interval $[1, n]$ so we assume that $1 \leq k \leq \frac{n-1}{2}$ and show

$$
\frac{h(k)}{h(k+1)} \leq 1
$$

Since $k \leq n-k$ and

$$
\frac{h(k)}{h(k+1)}=\left(\frac{k}{k+1}\right)^{k+\frac{1}{2}}\left(\frac{n-k+1}{n-k}\right)^{n-k+\frac{1}{2}}
$$

it is sufficient to prove that $\left(\frac{k}{k+1}\right)^{k+\frac{1}{2}}$ is an increasing function of $k$.

Let

$$
\tilde{h}(k)=\log \left(\frac{k}{k+1}\right)^{k+\frac{1}{2}}
$$

then $\left(\frac{k}{k+1}\right)^{k+\frac{1}{2}}$ is increasing if $\tilde{h}^{\prime}(k) \geq 0$.

$$
\begin{aligned}
\tilde{h}^{\prime}(k) & =\log \left(\frac{k}{k+1}\right)+\frac{k+\frac{1}{2}}{k(k+1)} \\
& =\log \left(\frac{k}{k+1}\right)+\frac{1}{2}\left(\frac{1}{k}+\frac{1}{k+1}\right) \\
& =-\int_{k}^{k+1} \frac{1}{t} d t+\frac{1}{2}\left(\frac{1}{k}+\frac{1}{k+1}\right)
\end{aligned}
$$

and by the convexity of the function $t \rightarrow \frac{1}{t}$ on $[0, \infty]$,

$$
\int_{k}^{k+1} \frac{1}{t} d t \leq \frac{1}{2}\left(\frac{1}{k}+\frac{1}{k+1}\right)
$$

so

$$
\tilde{h}^{\prime}(k) \geq 0
$$

as required, and

$$
h(k) \geq h(1)=h(n)=\left(\frac{n}{n+1}\right)^{n-\frac{1}{2}}
$$


Therefore

$$
\operatorname{Vol}_{n-1}(H \bigcap S) \geq \frac{\sqrt{n+1}}{(n-1) !}\left(\frac{n}{n+1}\right)^{n-\frac{1}{2}}
$$

the lower bound being attained when the slice is parallel to a facet of the simplex. 


\section{References}

1. K.M.Ball, Cube slicing in $R^{n}$, Proc. Amer. Math. Soc. 97 (3) (1986), 465-473.

2. K.M.Ball, Volumes of sections of cubes and related problems, Geometric aspects of functional analysis Israel seminar 1987-88, Springer-Verlag 1376 , 251-260.

3. K.M.Ball, Ellipsoids of maximal volume in convex bodies, Geometrie Dedicata 41 (1992), 241-250.

4. K.M.Ball, Volume ratios and a reverse isoperimetric inequality, J.London Math. Soc. (2) 44 (1991), 351-359.

5. J.J.M.Brands and G.Laman,Plane sections of a tetrahedron, American Math. Monthly 70 (1963), 338-339.

6. H.J.Brascamp and E.H.Lieb, Best constants in Young's inequality, its converse and its generalisation to more than three functions, Adv. in Math. 20 (1976) , 151-173. 
7. H.G.Eggleston, Plane sections of a tetrahedron, American Math.Monthly 70 (1963), 1108.

8. P.Filliman, The volume of duals and sections of polytopes, Mathematika 39 (1992), 67-80.

9. D.Hensley, Slicing the cube in $R^{n}$ and probability, Proc. Amer. Math. Soc. 73 (1) (1979), 95-100.

10. M.Meyer and A.Pajor, Sections of the unit ball of $\ell_{p}^{n}$, J. Func. Anal. 80 (1988), 109-123.

11. G.Pisier, The volume of convex bodies and Banach space geometry, Cambridge University Press (1989), Cambridge, pp. 3-4.

12. J.D.Vaaler, A geometric inequality with applications to linear forms, Pacific J.Math. 83 (1979), 543-553.

13. D.W.Walkup, A simplex with a large cross section, American Math.Monthly 75 (1968), 34-36. 